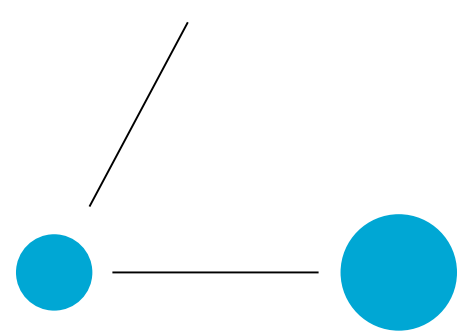

A N N A L E S

HENRI LEBESGUE

\title{
NO TEMPORAL DISTRIBUTIONAL LIMIT THEOREM FOR A. E. IRRATIONAL TRANSLATION
} UNE TRANSLATION IRRATIONNELE TYPIQUE NE SATISFAIT PAS DE THÉORĖME LIMITE TEMPOREL

ABSTRACT. - Bromberg and Ulcigrai constructed piecewise smooth functions on the circle such that the set of $\alpha$ for which the sum $\sum_{k=0}^{n-1} f(x+k \alpha$ mod 1) satisfies a temporal distributional limit theorem along the orbit of a.e. $x$ has Hausdorff dimension one. We show that the Lebesgue measure of this set is equal to zero.

RÉSUMÉ. - Bromberg et Ulcigrai ont construit des fonctions lisses par morceaux sur le cercle pour lesquelles l'ensemble des $\alpha$ tels que la somme $\sum_{k=0}^{n-1} f(x+k \alpha \bmod 1)$ satisfait un théorème limite temporel le long de l'orbite de presque tout $x$ est un ensemble de dimension de Hausdorff 1. Nous montrons que cet ensemble est de mesure nulle.

\section{Introduction and statement of main result}

\subsection{Background}

Suppose $T: X \rightarrow X$ is a map, $f: X \rightarrow \mathbb{R}$ is a function, and $x_{0} \in X$ is a fixed initial condition. We say that the $T$-ergodic sums $S_{n}=f\left(x_{0}\right)+f\left(T x_{0}\right)+\cdots+f\left(T^{n-1} x_{0}\right)$

2010 Mathematics Subject Classification: 37D25, 37D35.

DOI: https://doi.org/10.5802/ahl.4

(*) This work was partially supported by the ISF grants 199/14, 1149/18 and by the BSF grant 2016105. The authors thank Adam Kanigowski for simplifying the statement and the proof of Lemma 3.2. 
satisfy a temporal distributional limit theorem (TDLT) on the orbit of $x_{0}$, if there exists a non-constant real valued random variable $Y$, centering constants $A_{N} \in \mathbb{R}$ and scaling constants $B_{N} \rightarrow \infty$ s.t.

$$
\frac{S_{n}-A_{N}}{B_{N}} \underset{N \rightarrow \infty}{\longrightarrow} Y \text { in distribution, }
$$

when $n$ is sampled uniformly from $\{1, \ldots, N\}$ and $x_{0}$ is fixed. Equivalently, for every Borel set $E \subset \mathbb{R}$ s.t. $\mathbb{P}(Y \in \partial E)=0$,

$$
\frac{1}{N} \operatorname{Card}\left\{1 \leqslant n \leqslant N: \frac{S_{n}-A_{N}}{B_{N}} \in E\right\} \underset{N \rightarrow \infty}{\longrightarrow} \mathbb{P}(Y \in E) .
$$

We allow and expect $A_{N}, B_{N}, Y$ to depend on $T, f, x_{0}$.

Such limit theorems have been discovered for several zero entropy uniquely ergodic transformations, including systems where the more traditional spatial limit theorems, with $x_{0}$ is sampled from a measure on $X$, fail [Bec10, Bec11, ADDS15, DS17b, PS17, DS18]. Of particular interest are TDLT for

$$
R_{\alpha}:[0,1] \rightarrow[0,1], R_{\alpha}(x)=x+\alpha \bmod 1, f_{\beta}(x):=1_{[0, \beta)}(x)-\beta,
$$

because the $R_{\alpha}$-ergodic sums of $f_{\beta}$ along the orbit of $x$ represent the discrepancy of the sequence $x+n \alpha \bmod 1$ with respect to $[0, \beta)$ [Sch78, CK76, Bec10]. Another source of interest is the connection to the "deterministic random walk" [AK82, ADDS15].

The validity of the TDLT for $R_{\alpha}$ and $f_{\beta}$ depends on the diophantine properties of $\alpha$ and $\beta$. Recall that $\alpha \in(0,1)$ is badly approximable if for some $c>0,|q \alpha-p| \geqslant c /|q|$ for all irreducible fractions $p / q$. Equivalently, the digits in the continued fraction expansion of $\alpha$ are bounded [Khi63]. Say that $\beta \in(0,1)$ is badly approximable with respect to $\alpha$ if for some $C>0,|q \alpha-\beta-p|>C /|q|$ for all $p, q \in \mathbb{Z}, q \neq 0$. If $\alpha$ is badly approximable then every $\beta \in \mathbb{Q} \cap(0,1)$ is badly approximable with respect to $\alpha$. The recent paper [BU18] shows:

Theorem 1.1 (Bromberg-Ulcigrai [BU18]). - Suppose $\alpha$ is badly approximable and $\beta$ is badly approximable with respect to $\alpha$, e.g. $\beta \in \mathbb{Q} \cap(0,1)$. Then the $R_{\alpha^{-}}$ ergodic sums of $f_{\beta}$ satisfy a temporal distributional limit theorem with Gaussian limit on the orbit of every initial condition.

The set of badly approximable $\alpha$ has Hausdorff dimension one [Jar29], but Lebesgue measure zero [Khi24]. This leads to the following question: Is there a $\beta$ s.t. the $R_{\alpha}-$ ergodic sums of $f_{\beta}$ satisfy a temporal distributional limit theorem for a.e. $\alpha$ and a.e. initial condition?

In this paper we answer this question negatively.

\subsection{Main result}

To state our result in its most general form, we need the following terminology.

Let $\mathbb{T}:=\mathbb{R} / \mathbb{Z}$. We say that $f: \mathbb{T} \rightarrow \mathbb{R}$ is piecewise smooth if there exists a finite set $\mathfrak{S} \subset \mathbb{T}$ s.t. $f$ is continuously differentiable on $\mathbb{T} \backslash \mathfrak{S}$ and $\exists \psi: \mathbb{T} \rightarrow \mathbb{R}$ with bounded variation s.t. $f^{\prime}=\psi$ on $\mathbb{T} \backslash \mathfrak{S}$. For example: $f_{\beta}(x)=1_{[0, \beta)}(x)-\beta$ (take $\mathfrak{S}=\{0, \beta\}, \psi \equiv 0)$. We show: 
THEOREM 1.2. - Let $f$ be a piecewise smooth function of zero mean. Then there is a set of full measure $\mathcal{E} \subset \mathbb{T} \times \mathbb{T}$ s.t. if $(\alpha, x) \in \mathcal{E}$ then the $R_{\alpha}$-ergodic sums of $f$ do not satisfy a TDLT on the orbit of $x$.

The condition $\int_{\mathbb{T}} f=0$ is necessary: By Weyl's equidistribution theorem, for every $\alpha \notin \mathbb{Q}, f$ Riemann integrable s.t. $\int_{\mathbb{T}} f=1$, and $x_{0} \in \mathbb{T}, S_{n} / N \underset{N \rightarrow \infty}{\stackrel{\text { dist }}{\longrightarrow}} \mathrm{U}[0,1]$ as $n \sim \mathrm{U}(1, \ldots, N)$. See Section 1.4 for the notation.

This paper has a companion [DS17a] which gives a different proof of Theorem 1.2, in the special case $f(x)=\{x\}-\frac{1}{2}$. Unlike the proof given below, [DS17a] does not identify the set of $\alpha$ where the TDLT fails, but it does give more information on the different scaling limits for the distributions of $S_{n}, n \sim \mathrm{U}\left(1, \ldots, N_{k}\right)$ along different subsequences $N_{k} \rightarrow \infty$. [DS17a] also shows that if we randomize both $n$ and $\alpha$ by sampling $(n, \alpha)$ uniformly from $\{1, \ldots, N\} \times \mathbb{T}$, then $\left(S_{n}-\frac{1}{N} \sum_{k=1}^{N} S_{k}\right) / \sqrt{\ln N}$ converges in distribution to the Cauchy distribution.

The methods of [DS17a] are specific for $f(x)=\{x\}-\frac{1}{2}$, and we do not know how to apply them to other functions such as $f_{\beta}(x)=1_{[0, \beta)}(x)-\beta$.

\subsection{The structure of the proof}

Suppose $f$ is piecewise smooth and has mean zero.

We shall see below that if $f$ is continuous, then for a.e. $\alpha, f$ is an $R_{\alpha}$-coboundary, therefore $S_{n}$ are bounded, hence (1.1) cannot hold with $B_{N} \rightarrow \infty, Y$ non-constant. We remark that (1.1) does hold with $B_{N} \equiv 1, A_{N}=f\left(x_{0}\right), Y=$ distribution of minus the transfer function, but this is not a TDLT since no actual scaling is involved.

The heart of the proof is to show that if $f$ is discontinuous, then for a.e. $\alpha$, the temporal distributions of the ergodic sums have different asymptotic scaling behavior on different subsequences. The proof of this has three independent parts:

(1) A reduction to the case $f(x)=\sum_{m=1}^{d} b_{m} h\left(x+\beta_{m}\right), h(x):=\{x\}-\frac{1}{2}$.

(2) A proof that if $\mathcal{N} \subset \mathbb{N}$ has positive lower density, then there exists $M \geqslant 1$ s.t. the following set has full Lebesgue measure in $(0,1)$ :

$\mathcal{A}(\mathcal{N}, M):=\left\{\alpha \in(0,1): \begin{array}{l}\exists n_{k} \uparrow \infty, r_{k} \leqslant M \text { s.t. for all } k: \\ r_{k} q_{n_{k}} \in \mathcal{N}, a_{n_{k}+1} /\left(a_{1}+\cdots+a_{n_{k}}\right) \rightarrow \infty\end{array}\right\}$.

Here $a_{n}$ and $q_{n}$ are the partial quotients and principal denominators of $\alpha$, see Section 3.1.

(3) Construction of $\mathcal{N}=\mathcal{N}\left(b_{1}, \ldots, b_{d} ; \beta_{1}, \ldots, \beta_{d}\right) \subseteq \mathbb{N}$ with positive density, s.t. for every $\alpha \in \mathcal{A}(\mathcal{N}, M)$ and a.e. $x$, one can analyze the temporal distributions of the Birkhoff sums of $\sum_{m=1}^{d} b_{m} h\left(x+\beta_{m}\right)$.

\subsection{Notation}

$n \sim \mathrm{U}(1, \ldots, N)$ means that $n$ is a random variable taking values in $\{1, \ldots, N\}$, each with probability $\frac{1}{N}$. $\mathrm{U}[a, b]$ is the uniform distribution on $[a, b]$. Lebesgue's measure is denoted by mes. $\mathbb{N}=\{1,2,3, \ldots\}$ and $\mathbb{N}_{0}=\mathbb{N} \cup\{0\}$. If $x \in \mathbb{R}$, then $\|x\|:=\operatorname{dist}(x, \mathbb{Z})$ and $\{x\}$ is the unique number in $[0,1)$ s.t. $x \in\{x\}+\mathbb{Z}$. $\operatorname{Card}(\cdot)$ is the cardinality. If $\varepsilon>0$, then $a=b \pm \varepsilon$ means that $|a-b| \leqslant \varepsilon$. 


\section{Reduction to the case $f(x)=\sum_{m=1}^{d} b_{m} h\left(x+\beta_{m}\right)$}

Let $h(x)=\{x\}-\frac{1}{2}$, and let $\mathcal{G}$ denote the collection of all non-identically zero functions of the form $f(x)=\sum_{m=1}^{d} b_{m} h\left(x+\beta_{m}\right)$, where $d \in \mathbb{N}, b_{i}, \beta_{i} \in \mathbb{R}$. We explain how to reduce the proof of Theorem 1.2 from the case of a general piecewise smooth $f(x)$ to the case $f \in \mathcal{G}$.

The following proposition was proved in [DS17a]. Let $C(\mathbb{T})$ denote the space of continuous real-valued functions on $\mathbb{T}$ with the sup norm.

Proposition 2.1. - If $f(t)$ is differentiable on $\mathbb{T} \backslash\left\{\beta_{1}, \ldots, \beta_{d}\right\}$ and $f^{\prime}$ extends to a function with bounded variation on $\mathbb{T}$, then there are $d \in \mathbb{N}_{0}, b_{1}, \ldots, b_{d} \in \mathbb{R}$ s.t. for a.e. $\alpha \in \mathbb{T}$ there is $\varphi_{\alpha} \in C(\mathbb{T})$ s.t.

$$
f(x)=\sum_{i=1}^{d} b_{i} h\left(x+\beta_{i}\right)+\int_{\mathbb{T}} f(t) \mathrm{d} t+\varphi_{\alpha}(x)-\varphi_{\alpha}(x+\alpha) \quad\left(x \neq \beta_{1}, \ldots, \beta_{d}\right) .
$$

The following proposition was proved in [DS17b]. Let $(\Omega, \mathcal{B}, \mu)$ be a probability space, and let $T: \Omega \rightarrow \Omega$ be a probability preserving map.

Proposition 2.2. - Suppose $f=g+\varphi-\varphi \circ T \mu$-a.e. with $f, g, \varphi: \Omega \rightarrow \mathbb{R}$ measurable. If the ergodic sums of $g$ satisfy a TDLT along the orbit of a.e. $x$, then so do the ergodic sums of $f$.

These results show that if Theorem 1.2 holds for every $f \in \mathcal{G}$, then Theorem 1.2 holds for any discontinuous piecewise smooth function with zero mean. As for continuous piecewise smooth functions with zero mean, these are $R_{\alpha}$-cohomologous to $g \equiv 0$ for a.e. $\alpha$ because the $b_{i}$ in Proposition 2.1 must all vanish. Since the zero function does not satisfy the TDLT, continuous piecewise smooth functions do not satisfy a TDLT.

\section{The set $\mathcal{A}$ has full measure}

\subsection{Statement and plan of proof}

Let $\alpha$ be an irrational number, with continued fraction expansion denoted by $\left[a_{0} ; a_{1}, a_{2}, a_{3}, \ldots\right]:=a_{0}+\frac{1}{a_{1}+\ldots}, a_{0} \in \mathbb{Z}, a_{i} \in \mathbb{N}(i \geqslant 1)$. We call $a_{n}$ the quotients of $\alpha$. Let $p_{n} / q_{n}$ denote the principal convergents of $\alpha$, determined recursively by

$$
q_{n+1}=a_{n+1} q_{n}+q_{n-1}, \quad p_{n+1}=a_{n+1} p_{n}+p_{n-1}
$$

and $p_{0}=a_{0}, q_{0}=1 ; p_{1}=1+a_{1} a_{0}, q_{1}=a_{1}$. We call $q_{n}$ the principal denominators and $a_{i}$ the partial quotients of $\alpha$. Sometimes - but not always! - we will write $q_{k}=q_{k}(\alpha)$, $p_{k}=p_{k}(\alpha), a_{k}=a_{k}(\alpha)$.

Given $\mathcal{N} \subset \mathbb{N}$ and $M \geqslant 1$, let $\mathcal{A}=\mathcal{A}(\mathcal{N}, M) \subset(0,1)$ denote the set of irrational $\alpha \in(0,1)$ s.t. for some subsequence $n_{k} \uparrow \infty$,

$$
\exists r_{k} \leqslant M \text { s.t. } r_{k} q_{n_{k}} \in \mathcal{N}, \frac{a_{n_{k}+1}}{\left(a_{0}+\cdots+a_{n_{k}}\right)} \underset{k \rightarrow \infty}{\longrightarrow} \infty .
$$

The lower density of $\mathcal{N}$ is $d(\mathcal{N}):=\liminf \frac{1}{N} \operatorname{Card}(\mathcal{N} \cap[1, N])$. The purpose of this section is to prove: 
TheOREM 3.1. - If a set $\mathcal{N}$ has positive lower density, then there exists $M$ such that $\mathcal{A}(\mathcal{N}, M)$ has full Lebesgue measure in $(0,1)$.

The proof consists of the following three lemmas:

Lemma 3.2. - For almost all $\alpha$ there is $n_{0}=n_{0}(\alpha)$ s.t. if $k \geqslant n_{0}$ and $a_{k+1}>$ $\frac{1}{4} k(\ln k)(\ln \ln k)$, then $a_{k+1} /\left(a_{1}+\cdots+a_{k}\right) \geqslant \frac{1}{8} \ln \ln k$.

Lemma 3.3. - Suppose $\alpha \in(0,1) \backslash \mathbb{Q}$ and $(p, q) \in \mathbb{N}_{0} \times \mathbb{N}$ satisfy $\operatorname{gcd}(p, q)=1$ and $|q \alpha-p| \leqslant \frac{1}{q L}$ where $L \geqslant 4$. Then there exists $k$ s.t. $q=q_{k}(\alpha)$ and $a_{k+1}(\alpha) \geqslant \frac{1}{2} L$.

Lemma 3.4. - Suppose $\psi: \mathbb{R}_{+} \rightarrow \mathbb{R}$ is a non-decreasing function s.t.

$$
\sum_{n} \frac{1}{n \psi(n)}=\infty
$$

Suppose $\mathcal{N} \subset \mathbb{N}$ has positive lower density. For all $M$ sufficiently large, for a.e. $\alpha \in(0,1)$ there are infinitely many pairs $(m, n) \in \mathbb{N}_{0} \times \mathbb{N}$ s.t. $n \in \mathcal{N}, \operatorname{gcd}(m, n) \leqslant M$, and $|n \alpha-m| \leqslant \frac{1}{n \psi(n)}$.

Remark 3.5. - By the monotonicity of $\psi$, if $e^{k-1}<n<e^{k}$ then $\psi\left(e^{k-1}\right) \leqslant$ $\psi(n) \leqslant \psi\left(e^{k}\right)$. Hence (3.2) holds iff $\sum_{k} \frac{1}{\psi\left(e^{k}\right)}=\infty$.

Remark 3.6. - If $\mathcal{N}=\mathbb{N}$, then Lemma 3.4 holds with $M=1$ by the classical Khinchine Theorem. We do not know if Lemma 3.4 holds with $M=1$ for every set $\mathcal{N}$ with positive lower density.

Proof of Theorem 3.1 given Lemmas 3.2-3.4. - We apply these lemmas with $\psi(t)=c(\ln t)(\ln \ln t)(\ln \ln \ln t)$ and $c>1 / \ln \left(\frac{1+\sqrt{5}}{2}\right)$.

Fix $M>1$ as in Lemma 3.4. Then $\exists \Omega \subset(0,1)$ of full measure s.t. for every $\alpha \in \Omega$ there are infinitely many $(m, n) \in \mathbb{N}_{0} \times \mathbb{N}$ as follows. Let $m^{*}:=m / \operatorname{gcd}(m, n)$, $n^{*}:=n / \operatorname{gcd}(m, n), p:=\operatorname{gcd}(m, n)$, then

(1) $p n^{*} \in \mathcal{N}, p \leqslant M,\left|n^{*} \alpha-m^{*}\right|=\frac{|n \alpha-m|}{p} \leqslant \frac{1}{n^{*} \psi\left(n^{*}\right)}\left(\because n^{*} \leqslant n\right)$;

(2) $\exists k$ s.t. $n^{*}=q_{k}(\alpha)$ and $a_{k+1}(\alpha) \geqslant \frac{1}{2} \psi\left(q_{k}\right)(\because$ Lemma 3.3). By its recursive definition, $q_{k} \geqslant k$-th Fibonacci number $\geqslant \frac{1}{3}\left(\frac{1+\sqrt{5}}{3}\right)^{k}$. So for all $k$ large enough, $a_{k+1}(\alpha) \geqslant \frac{1}{2} \psi\left(q_{k}\right)>\frac{1}{4} k(\ln k)(\ln \ln k)$;

(3) $a_{k+1} /\left(a_{1}+\cdots+a_{k}\right) \geqslant \frac{1}{8} \ln \ln k \rightarrow \infty(\because$ Lemma 3.2).

So every $\alpha \in \Omega$ belongs to $\mathcal{A}=\mathcal{A}(\mathcal{N}, M)$, and $\mathcal{A}$ has full measure.

Next we prove Lemmas 3.2-3.4.

\subsection{Proof of Lemma 3.2}

By [DV86], for almost every $\alpha$

$$
\frac{\left(a_{1}+\cdots+a_{k+1}\right)-\max _{j \leqslant k+1} a_{j}}{k \ln k} \rightarrow \frac{1}{\ln 2}<2 .
$$

So if $k$ is large enough, and $a_{k+1}>\frac{1}{4} k(\ln k)(\ln \ln k)$ then

$$
\max _{j \leqslant k+1} a_{j}=a_{k+1}, \quad \frac{a_{1}+\cdots+a_{k}}{k \ln k} \leqslant 2, \text { and } \frac{a_{k+1}}{a_{1}+\cdots+a_{k}}>\frac{1}{8} \ln \ln k .
$$




\subsection{Proof of Lemma 3.3}

For every $(p, q)$ as in the lemma, $|q \alpha-p|<\frac{1}{2 q}$. A classical result in the theory of continued fractions [Khi63, Theorem 19] says that in this case $\exists k$ s.t. $q=q_{k}(\alpha), p=$ $p_{k}(\alpha)$.

To estimate $a_{k+1}=a_{k+1}(\alpha)$ we recall the following facts, valid for the principal denominators of any irrational $\alpha \in(0,1)$ [Khi63]:

(1) $\left|q_{k} \alpha-p_{k}\right|>\frac{1}{q_{k}+q_{k+1}}$;

(2) $q_{k+1}+q_{k}<\left(a_{k+1}+2\right) q_{k}$, whence by (a) $a_{k+1}>\frac{1}{q_{k}\left|q_{k} \alpha-p_{k}\right|}-2$.

In our case, $\left|q_{k} \alpha-p_{k}\right|=|q \alpha-p| \leqslant \frac{1}{q_{k} L}$, so $a_{k+1}>L-2 \geqslant \frac{L}{2}$.

\subsection{Preparations for the proof of Lemma 3.4}

Let $(\Omega, \mathcal{F}, \mathbb{P})$ be a probability space, and $A_{k} \in \mathcal{F}$ be measurable events. Given $D>1$, we say that $A_{k}$ are $D$-quasi-independent, if

$$
\mathbb{P}\left(A_{k_{1}} \cap A_{k_{2}}\right) \leqslant D \mathbb{P}\left(A_{k_{1}}\right) \mathbb{P}\left(A_{k_{2}}\right) \text { for all } k_{1} \neq k_{2} \text {. }
$$

The following proposition is a slight variation on Sullivan's Borel-Cantelli Lemma from $([$ Sul82]):

Proposition 3.7. - For every $D \geqslant 1$ there exists a constant $\delta(D)>0$ such that the following holds in any probability space:

(a) If $A_{k}$ are $D$-quasi-independent measurable events s.t. $\lim _{k \rightarrow \infty} \mathbb{P}\left(A_{k}\right)=0$ but $\sum_{k} \mathbb{P}\left(A_{k}\right)=\infty$, then $\mathbb{P}\left(A_{k}\right.$ occurs infinitely often $) \geqslant \delta(D)$.

(b) The quasi-independence assumption in (a) can be weakened to the assumption that for some $r \in \mathbb{N}, \mathbb{P}\left(A_{k_{1}} \cap A_{k_{2}}\right) \leqslant D \mathbb{P}\left(A_{k_{1}}\right) \mathbb{P}\left(A_{k_{2}}\right)$ for all $\left|k_{2}-k_{1}\right| \geqslant r$.

(c) One can take $\delta(D)=\frac{1}{2 D}$.

Proof. - Since $\mathbb{P}\left(A_{k}\right) \rightarrow 0$ but $\sum \mathbb{P}\left(A_{k}\right)=\infty$, there is an increasing sequence $N_{j}$ such that $\lim _{j \rightarrow \infty} \sum_{k=N_{j}+1}^{N_{j+1}} \mathbb{P}\left(A_{k}\right)=\frac{1}{D}$.

Let $B_{j}$ be the event that at least one of events $\left\{A_{k}\right\}_{k=N_{j}+1}^{N_{j+1}}$ occurs. Since $B_{j}=$ $\biguplus_{k=N_{j+1}}^{N_{j}+1}\left(A_{k} \backslash \bigcup_{j=N_{j}+1}^{k-1} A_{j}\right)$,

$$
\begin{aligned}
\mathbb{P}\left(B_{j}\right) & \geqslant \sum_{k=N_{j}+1}^{N_{j+1}} \mathbb{P}\left(A_{k}\right)-\sum_{N_{j}+1 \leqslant k_{1}<k_{2} \leqslant N_{j+1}} \mathbb{P}\left(A_{k_{1}} \cap A_{k_{2}}\right) \\
& \geqslant \sum_{k=N_{j}+1}^{N_{j+1}} \mathbb{P}\left(A_{k}\right)-D \sum_{N_{j}+1 \leqslant k_{1}<k_{2} \leqslant N_{j+1}} \mathbb{P}\left(A_{k_{1}}\right) \mathbb{P}\left(A_{k_{2}}\right) \\
& \geqslant \sum_{k=N_{j}+1}^{N_{j+1}} \mathbb{P}\left(A_{k}\right)-\frac{D}{2}\left(\sum_{k=N_{j}+1}^{N_{j+1}} \mathbb{P}\left(A_{k}\right)\right)^{2} .
\end{aligned}
$$

Since $\lim _{j \rightarrow \infty} \sum_{k=N_{j}+1}^{N_{j+1}} \mathbb{P}\left(A_{k}\right)=\frac{1}{D}$ and $D \geqslant 1, \liminf \mathbb{P}\left(B_{j}\right) \geqslant \frac{1}{2 D}$.

Let $E$ denote the event that $A_{j}$ happens infinitely often. $E$ is also the event that $B_{j}$ happens infinitely often, therefore $E=\bigcap_{n=1}^{\infty} \bigcup_{j=n+1}^{\infty} B_{j}$. In a probability space, 
the measure of a decreasing intersection of sets is the limit of the measure of these sets. So $\mathbb{P}(E) \geqslant \liminf \mathbb{P}\left(B_{j}\right) \geqslant \frac{1}{2 D}$, proving (a) and (c).

Part (b) follows from part (a) by applying it to the sets $\left\{A_{k r+\ell}\right\}$ where $0 \leqslant \ell \leqslant r-1$ is chosen to get $\sum_{k} \mathbb{P}\left(A_{k r+\ell}\right)=\infty$.

The multiplicity of a collection of measurable sets $\left\{E_{k}\right\}$ is defined to be the largest $K$ s.t. there are $K$ different $k_{i}$ with $\mathbb{P}\left(\bigcap_{i=1}^{K} E_{k_{i}}\right)>0$.

Proposition 3.8. - Let $E_{k}$ be measurable sets in a finite measure space. If the multiplicity of $\left\{E_{k}\right\}$ is less than $K$, then

$$
\operatorname{mes}\left(\bigcup_{k} E_{k}\right) \geqslant \frac{1}{K} \sum_{k} \operatorname{mes}\left(E_{k}\right) \text {. }
$$

Proof. $-1 \bigcup_{i} E_{i} \geqslant \frac{1}{K} \sum_{i} 1_{E_{i}}$ almost everywhere.

Proposition 3.9. - For every non-empty open interval $I \subset[0,1]$,

Card $\left\{(m, n) \in\{0, \ldots, N\}^{2}: \frac{m}{n} \in I, \operatorname{gcd}(m, n)=1\right\} \sim 3 \operatorname{mes}(I) N^{2} / \pi^{2}$, as $N \rightarrow \infty$.

Proof. - This classical fact due to Dirichlet follows from the inclusion-exclusion principle and the identity $\zeta(2)=\pi^{2} / 6$, see [HW08, Theorem 459].

Proposition 3.10. - Suppose $\alpha=\left[0 ; a_{1}, a_{2}, \ldots\right]$ and $\bar{\alpha}=\left[0 ; a_{\ell+1}, a_{\ell+2}, \ldots\right]$. Then the principal convergents $\bar{p}_{\bar{\ell}} / \bar{q}_{\bar{\ell}}$ of $\bar{\alpha}$ and the principal convergents $p_{\ell} / q_{\ell}$ of $\alpha$ are related by

$$
\left(\begin{array}{cc}
p_{l+\bar{l}} & p_{l+\bar{l}+1} \\
q_{l+\bar{l}} & q_{l+\bar{l}+1}
\end{array}\right)=\left(\begin{array}{cc}
p_{l-1} & p_{l} \\
q_{l-1} & q_{l}
\end{array}\right)\left(\begin{array}{cc}
\bar{p}_{\bar{l}} & \bar{p}_{\bar{l}+1} \\
\bar{q}_{\bar{l}} & \bar{q}_{\bar{l}+1}
\end{array}\right) .
$$

Proof. - Since $a_{0}=0$, the recurrence relations for $p_{n} / q_{n}$ imply

$$
\left(\begin{array}{cc}
p_{n} & p_{n+1} \\
q_{n} & q_{n+1}
\end{array}\right)=\left(\begin{array}{cc}
p_{n-1} & p_{n} \\
q_{n-1} & q_{n}
\end{array}\right)\left(\begin{array}{cc}
0 & 1 \\
1 & a_{n+1}
\end{array}\right),\left(\begin{array}{cc}
p_{0} & p_{1} \\
q_{0} & q_{1}
\end{array}\right)=\left(\begin{array}{cc}
0 & 1 \\
1 & a_{1}
\end{array}\right) .
$$

So

$$
\left(\begin{array}{cc}
p_{n} & p_{n+1} \\
q_{n} & q_{n+1}
\end{array}\right)=\left(\begin{array}{cc}
0 & 1 \\
1 & a_{1}
\end{array}\right) \cdots\left(\begin{array}{cc}
0 & 1 \\
1 & a_{n+1}
\end{array}\right)
$$

It follows that

$$
\left(\begin{array}{cc}
p_{l+\bar{l}} & p_{l+\bar{l}+1} \\
q_{l+\bar{l}} & q_{l+\bar{l}+1}
\end{array}\right)=\left(\begin{array}{cc}
p_{l-1} & p_{l} \\
q_{l-1} & q_{l}
\end{array}\right)\left(\begin{array}{cc}
\bar{p}_{\bar{l}} & \bar{p}_{\bar{l}+1} \\
\bar{q}_{\bar{l}} & \bar{q}_{\bar{l}+1}
\end{array}\right)
$$

where $\bar{p}_{i} / \bar{q}_{i}$ are the principal convergents of $\bar{\alpha}:=\left[0 ; a_{l+1}, a_{l+2}, \ldots\right]$.

\subsection{Proof of Lemma 3.4}

Without loss of generality, $\lim _{t \rightarrow \infty} \psi(t)=\infty$, otherwise replace $\psi(t)$ by the bigger monotone function $\psi(t)+\ln t$. 
Fix $M>1$, to be determined later. Let

$$
\begin{aligned}
\Omega_{k} & :=\left\{(m, n) \in \mathbb{N}^{2}: n \in \mathcal{N}, n \in\left[e^{k-1}, e^{k}\right], 0<m<n, \operatorname{gcd}(m, n) \leqslant M\right\}, \\
A_{m, n, k} & :=\left\{\alpha \in \mathbb{T}:|n \alpha-m| \leqslant \frac{1}{e^{k} \psi\left(e^{k}\right)}\right\}, \\
\mathcal{A}_{k} & :=\bigcup_{(m, n) \in \Omega_{k}} A_{m, n, k}, \\
\mathcal{A} & :=\left\{\alpha \in \mathbb{T}: \alpha \text { belongs to infinitely many } \mathcal{A}_{k}\right\} .
\end{aligned}
$$

The lemma is equivalent to saying that $\mathcal{A}$ has full Lebesgue measure for a suitable choice of $M$.

We will prove a slightly different statement. Fix $\varepsilon>0$ small. Given an non-empty interval $I \subset[\varepsilon, 1-\varepsilon]$, let

$$
\begin{aligned}
\Omega_{k}(I) & :=\left\{(m, n) \in \Omega_{k}: \frac{m}{n} \in I\right\}, \\
\mathcal{A}_{k}(I) & :=\bigcup_{(m, n) \in \Omega_{k}(I)} A_{m, n, k}, \\
\mathcal{A}(I) & :=\left\{\alpha \in \mathbb{T}: \alpha \text { belongs to infinitely many } \mathcal{A}_{k}(I)\right\} .
\end{aligned}
$$

We will prove that there exists a positive constant $\delta=\delta(\varepsilon, M)$ s.t. for all intervals $I \subset[\varepsilon, 1-\varepsilon], \operatorname{mes}(\mathcal{A}(I) \cap I) \geqslant \delta \operatorname{mes}(I)$. It then follows by a standard density point $\operatorname{argument}$ (see below) that $\mathcal{A} \cap[\varepsilon, 1-\varepsilon]$ has full measure. Since $\varepsilon$ is arbitrary, the lemma is proved.

Claim 1. - There exists $K=K(\varepsilon)$ such that for every $k>K$, the multiplicity of $\left\{A_{m, n, k}\right\}_{(m, n) \in \Omega_{k}(I)}$ is uniformly bounded by $M$.

Proof. - Suppose $\left(m_{i}, n_{i}\right) \in \Omega_{k}(I)(i=1,2)$ and $A_{m_{1}, n_{1}, k} \cap A_{m_{2}, n_{2}, k} \neq \varnothing$. Then there is $\alpha$ s.t. $\left|n_{i} \alpha-m_{i}\right| \leqslant \delta_{k}:=\frac{1}{e^{k} \psi\left(e^{k}\right)}(i=1,2)$. Choose $K=K(\varepsilon)$ so large that $k>K \Rightarrow \delta_{k}<\frac{\varepsilon}{4 e^{k}}$.

If $k>K$, then $\alpha \geqslant \frac{m_{i}}{n_{i}}-\delta_{k}>\min I-\frac{\varepsilon}{2}>\frac{\varepsilon}{2}$. Let $r_{i}:=\operatorname{gcd}\left(m_{i}, n_{i}\right)$ and $\left(n_{i}^{*}, m_{i}^{*}\right):=$ $\frac{1}{r_{i}}\left(n_{i}, m_{i}\right)$. Then $\left|n_{i}^{*} \alpha-m_{i}^{*}\right| \leqslant \delta_{k}$ and $m_{i}^{*} \leqslant n_{i}^{*} \leqslant n_{i} \leqslant e^{k}$, so $\left|n_{2}^{*} m_{1}^{*}-n_{1}^{*} m_{2}^{*}\right|=$ $\frac{1}{\alpha}\left|m_{1}^{*}\left(n_{2}^{*} \alpha-m_{2}^{*}\right)-m_{2}^{*}\left(n_{1}^{*} \alpha-m_{1}^{*}\right)\right| \leqslant \frac{2 e^{k} \delta_{k}}{\varepsilon / 2}<1$. So $n_{2}^{*} m_{1}^{*}=n_{1}^{*} m_{2}^{*}$. Since $\operatorname{gcd}\left(n_{i}^{*}, m_{i}^{*}\right)=$ $1,\left(n_{1}^{*}, m_{1}^{*}\right)=\left(n_{2}^{*}, m_{2}^{*}\right)$. It follows that $\left(n_{2}, m_{2}\right) \in\left\{\left(r n_{1}^{*}, r m_{1}^{*}\right): r=1, \ldots, M\right\}$. So the multiplicity of $\left\{A_{m, n, k}\right\}_{(m, n) \in \Omega_{k}(I)}$ is uniformly bounded by $M$.

Claim 2. - Let $d(\mathcal{N}):=\liminf \frac{1}{N} \operatorname{Card}(\mathcal{N} \cap[1, N])>0$, then there exists $M=M(\mathcal{N})$ and $\widetilde{K}=\widetilde{K}(\varepsilon, \mathcal{N},|I|)$ s.t. for all $k>\widetilde{K}$,

$$
\frac{d(\mathcal{N}) \operatorname{mes}(I)}{4 M \psi\left(e^{k}\right)} \leqslant \operatorname{mes}\left(\mathcal{A}_{k}(I)\right) \leqslant \frac{6 \operatorname{mes}(I)}{\psi\left(e^{k}\right)} .
$$

In particular, $\operatorname{mes}\left(\mathcal{A}_{k}(I)\right) \underset{k \rightarrow \infty}{\longrightarrow} 0$ and $\sum \operatorname{mes}\left(\mathcal{A}_{k}(I)\right)=\infty$.

Proof. - $\operatorname{mes}\left(A_{m, n, k}\right)=\operatorname{mes}\left(\left[\frac{m}{n}-r_{m, n}, \frac{m}{n}+r_{m, n}\right]\right)=2 r_{m, n}$ where $r_{m, n}=\frac{1}{n e^{k} \psi\left(e^{k}\right)}$. Since $n \in\left[e^{k-1}, e^{k}\right]$,

$$
\frac{\operatorname{Card}\left(\Omega_{k}(I)\right)}{M e^{2 k} \psi\left(e^{k}\right)} \leqslant \operatorname{mes}\left(\mathcal{A}_{k}(I)\right) \leqslant \frac{e \operatorname{Card}\left(\Omega_{k}(I)\right)}{e^{2 k} \psi\left(e^{k}\right)},
$$


where the lower bound uses Claim 1 and Proposition 3.8.

$\operatorname{Card}\left(\Omega_{k}(I)\right)$ satisfies the bounds $A-B \leqslant \operatorname{Card}\left(\Omega_{k}(I)\right) \leqslant A$ where

$$
\begin{aligned}
& A:=\operatorname{Card}\left\{(m, n): n \in \mathcal{N}, n \in\left[e^{k-1}, e^{k}\right], \frac{m}{n} \in I\right\} \\
& B:=\operatorname{Card}\left\{(m, n): n \in \mathcal{N}, n \in\left[e^{k-1}, e^{k}\right], \frac{m}{n} \in I, \operatorname{gcd}(m, n) \geqslant M\right\} .
\end{aligned}
$$

Choose $\widetilde{K}=\widetilde{K}(\varepsilon, \mathcal{N},|I|)>K(\varepsilon)$ s.t. for all $k>\widetilde{K}$

(1) $\operatorname{Card}\left\{n \in \mathcal{N}: 0 \leqslant n \leqslant e^{k}\right\} \geqslant \frac{1}{\sqrt{2}} d(\mathcal{N})$

(2) $\operatorname{Card}\left\{n \in\left[e^{k-1}, e^{k}\right] \cap \mathbb{N}: p \mid n\right\} \leqslant 2\left(e^{k}-e^{k-1}\right) / p$ for all $p \geqslant 1$;

(3) For all $n>e^{\widetilde{K}-1}, p \geqslant 1$,

$$
\frac{n}{p \sqrt{2}} \operatorname{mes}(I) \leqslant \operatorname{Card}\left\{m \in \mathbb{N}: \frac{m}{n} \in I, p \mid m\right\} \leqslant \frac{2 n}{p} \operatorname{mes}(I) .
$$

If $k>\widetilde{K}$, then $\frac{1}{2} d(\mathcal{N}) e^{2 k} \operatorname{mes}(I) \leqslant A \leqslant 2 e^{2 k} \operatorname{mes}(I)$ and

$$
\begin{aligned}
B & \leqslant \sum_{p=M}^{\infty} \operatorname{Card}\left\{(m, n): n \in\left[e^{k-1}, e^{k}\right], \frac{m}{n} \in I, p|m, p| n\right\} \\
& \leqslant \sum_{p=M}^{\infty} \frac{2\left(e^{k}-e^{k-1}\right)}{p} \cdot \frac{2 e^{k} \operatorname{mes}(I)}{p}<4 e^{2 k} \operatorname{mes}(I) \sum_{p=M}^{\infty} \frac{1}{p^{2}} \\
& \leqslant \frac{1}{4} d(\mathcal{N}) e^{2 k} \operatorname{mes}(I), \quad \text { provided we choose } M \text { s.t. } \sum_{p=M}^{\infty} p^{-2}<\frac{1}{16} d(\mathcal{N}) .
\end{aligned}
$$

Together we get $\frac{1}{4} d(\mathcal{N}) e^{2 k} \operatorname{mes}(I) \leqslant \operatorname{Card}\left(\Omega_{k}(I)\right) \leqslant 2 e^{2 k} \operatorname{mes}(I)$. The claim now follows from (3.5).

Claim 3. - There exists $D=D(\mathcal{N}, M), r=r(M)$, and $\hat{K}=\hat{K}(\varepsilon, \mathcal{N}, I)$ s.t. for all $k_{1}, k_{2}>\hat{K}$ s.t. $\left|k_{1}-k_{2}\right|>r(M)$,

$$
\operatorname{mes}\left(\mathcal{A}_{k_{1}}(I) \cap \mathcal{A}_{k_{2}}(I) \mid I\right) \leqslant D \operatorname{mes}\left(\mathcal{A}_{k_{1}}(I) \mid I\right) \operatorname{mes}\left(\mathcal{A}_{k_{2}}(I) \mid I\right)
$$

Proof. - By Claim 2, if $k_{1}, k_{2}$ are large enough, then

$$
\operatorname{mes}\left(\mathcal{A}_{k_{1}}(I) \mid I\right) \operatorname{mes}\left(\mathcal{A}_{k_{2}}(I) \mid I\right) \geqslant\left(\frac{d(\mathcal{N})}{5 M}\right)^{2} \frac{1}{\psi\left(e^{k_{1}}\right) \psi\left(e^{k_{2}}\right)},
$$

where we put 5 instead of 4 in the denominator to deal with edge effects arising from $\operatorname{mes}\left(\mathcal{A}_{k}(I) \backslash I\right)=O\left(\frac{1}{e^{k} \psi\left(e^{k}\right)}\right)$.

To prove the claim, it remains to bound mes $\left(\mathcal{A}_{k_{1}}(I) \cap \mathcal{A}_{k_{2}}(I) \mid I\right)$ from above by $\frac{\text { const }}{R_{1} R_{2}}$, where $R_{i}:=\psi\left(e^{k_{i}}\right)$.

A cylinder is a set of the form

$$
\llbracket a_{1}, \ldots, a_{n} \rrbracket=\left\{\alpha \in(0,1) \backslash \mathbb{Q}: a_{i}(\alpha)=a_{i}(1 \leqslant i \leqslant n)\right\} .
$$

Equivalently, $\alpha \in \llbracket a_{1}, \ldots, a_{n} \rrbracket$ iff $\alpha$ has an infinite continued fraction expansion of the form $\alpha=\left[0 ; a_{1}, \ldots, a_{n}, *, *, \ldots\right]$. 
Our plan is to cover $\mathcal{A}_{k_{i}}(I)$ by unions of cylinders of total measure $O\left(1 / R_{i}\right)$, and then use the following well-known fact: There is a constant $G>1$ s.t. for any $\left(a_{1}, \ldots, a_{n}, b_{1}, \ldots, b_{m}\right) \in \mathbb{N}^{n+m}$,

$$
G^{-1} \leqslant \frac{\operatorname{mes} \llbracket a_{1}, \ldots, a_{n} ; b_{1}, \ldots, b_{m} \rrbracket}{\operatorname{mes} \llbracket a_{1}, \ldots, a_{n} \rrbracket \operatorname{mes} \llbracket b_{1}, \ldots, b_{m} \rrbracket} \leqslant G .
$$

This is because the invariant measure $\frac{1}{\ln 2} \frac{d x}{1+x}$ of $T:(0,1) \rightarrow(0,1), T(x)=\left\{\frac{1}{x}\right\}$ (the Gauss map) is a Gibbs-Markov measure, thanks to the bounded distortion of $T$, see [ADU93, Section 2].

To cover $\mathcal{A}_{k_{i}}(I)$ by cylinders, it is enough to cover $A_{m, n, k_{i}}$ by cylinders for every $(m, n) \in \Omega_{k_{i}}(I)$. Suppose $\alpha \in A_{m, n, k_{i}}$. Then $r:=\operatorname{gcd}(m, n) \leqslant M$ and $\left(m^{*}, n^{*}\right):=$ $\frac{1}{r}(m, n)$ satisfies

$$
\operatorname{gcd}\left(m^{*}, n^{*}\right)=1, n^{*} \in \bigcup_{\left|k_{i}^{*}-k_{i}\right| \leqslant \ln M}\left[e^{k_{i}^{*}-1}, e^{k_{i}^{*}}\right],\left|n^{*} \alpha-m^{*}\right|<\frac{1}{n^{*} R_{i}} .
$$

Assume $k_{i}$ is so large that $R_{i}=\psi\left(e^{k_{i}}\right) \geqslant 4$. Then Lemma 3.3 gives $a_{l_{i}+1}>\frac{R_{i}}{2}$. Thus $\mathcal{A}_{k_{i}}(I) \subset \mathcal{C}_{k_{i}}\left(I, R_{i}\right)$ where

$$
\mathcal{C}_{k}(I, R):=\bigcup_{k^{*} \in[k-\ln M, k]}\left\{\alpha \in(0,1) \backslash \mathbb{Q}: \exists \ell \text { s.t. } \begin{array}{l}
q_{\ell}(\alpha) \in\left[e^{k^{*}-1}, e^{k^{*}}\right] \\
p_{\ell}(\alpha) / q_{\ell}(\alpha) \in R / 2
\end{array}\right\} .
$$

This is a union of cylinders, because $q_{\ell}(\alpha), p_{\ell}(\alpha), a_{\ell+1}(\alpha)$ are constant on cylinders of length $\ell+1$.

We claim that for some $c^{*}(M)$ which only depends on $M$, for all $k_{i}$ large enough,

$$
\operatorname{mes}\left(\mathcal{C}_{k_{i}}\left(I, R_{i}\right)\right) \leqslant \frac{c^{*}(M) \operatorname{mes}(I)}{R_{i}} .
$$

Every rational $\frac{m}{n} \in(0,1)$ has two finite continued fraction expansions: $\left[0 ; a_{1}, \ldots, a_{\ell}\right]$ and $\left[0 ; a_{1}, \ldots, a_{\ell}-1,1\right]$ with $a_{\ell}>1$. We write $\ell=\ell\left(\frac{m}{n}\right)$ and $a_{i}=a_{i}\left(\frac{m}{n}\right)$. With this notation

$$
\begin{aligned}
\mathcal{C}_{k_{i}}\left(I, R_{i}\right)= & \bigcup_{\substack{k_{i}^{*} \in\left[k_{i}-\ln M, k_{i}\right] \\
n \in\left[e^{k_{i}^{*}-1}, e^{k_{i}^{*}}\right]}} \bigcup_{\substack{\operatorname{gcd}(m, n)=1 \\
m / n \in I}} \bigcup_{b>R_{i} / 2} \\
\cup & \llbracket\left[a_{1}\left(\frac{m}{n}\right), \ldots, a_{\ell\left(\frac{m}{n}\right)}\left(\frac{m}{n}\right), b \rrbracket\right. \\
\cup & \llbracket a_{1}\left(\frac{m}{n}\right), \ldots, a_{\ell\left(\frac{m}{n}\right)}\left(\frac{m}{n}\right)-1,1, b \rrbracket .
\end{aligned}
$$

We have $\llbracket a_{1}, \ldots, a_{\ell} \rrbracket=\left(\frac{p_{\ell}+p_{\ell-1}}{q_{\ell}+q_{\ell-1}}, \frac{p_{\ell}}{q_{\ell}}\right)$ or $\left(\frac{p_{\ell}}{q_{\ell}}, \frac{p_{\ell}+p_{\ell-1}}{q_{\ell}+q_{\ell-1}}\right)$, depending on the parity of $\ell$, see for instance [Khi63]. Since $\left|p_{\ell} q_{\ell-1}-p_{\ell-1} q_{\ell}\right|=1$ and $q_{\ell+1}=a_{\ell+1} q_{\ell}+q_{\ell-1}$, we have $\operatorname{mes}\left(\llbracket a_{1}, \ldots, a_{\ell}, b \rrbracket\right)=\frac{1}{q_{\ell+1}\left(q_{\ell+1}+q_{\ell}\right)}=\frac{1}{\left(b q_{\ell}+q_{\ell-1}\right)\left((b+1) q_{\ell}+q_{\ell-1}\right)} \leqslant \frac{1}{b(b+1) q_{\ell}^{2}}$, leading to

$$
\begin{aligned}
& \operatorname{mes}\left(\mathcal{C}_{k_{i}}\left(I, R_{i}\right)\right) \leqslant \sum_{k_{i}^{*} \in\left[k_{i}-\ln M, k_{i}\right]} \sum_{n \in\left[e^{k_{i}^{*}-1}, e^{\left.k_{i}^{*}\right]}\right.} \sum_{\substack{\operatorname{gcd}(m, n)=1 \\
m / n \in I}} \sum_{b>R_{i} / 2} \frac{2}{n^{2} b(b+1)} \\
& \leqslant \frac{8 \ln M}{e^{2\left(k_{i}-1-\ln M\right)} R_{i}} \sum_{n=1}^{e^{k_{i} M}} \#\left\{m \in \mathbb{N}: \frac{m}{n} \in I, \operatorname{gcd}(m, n)=1\right\} \leqslant \frac{c^{*}(M)}{R_{i}} \operatorname{mes}(I)
\end{aligned}
$$

where $c^{*}(M)$ only depends on $M$. The last step uses Proposition 3.9. 
Next we cover $\mathcal{A}_{k_{1}}(I) \cap \mathcal{A}_{k_{2}}(I)$ by cylinders. Suppose without loss of generality that $k_{2}>k_{1}$. Arguing as before one sees that if

$$
k_{2}>k_{1}+\ln M+1,
$$

then $\mathcal{A}_{k_{1}}(I) \cap \mathcal{A}_{k_{2}}(I)$ can be covered by sets $\llbracket a_{1}, \ldots, a_{\ell}, b, \bar{a}_{1}, \ldots, \bar{a}_{\bar{l}}, \bar{b} \rrbracket$ as follows: The convergents $p_{i} / q_{i}$ of (every) $\alpha$ in $\llbracket a_{1}, \ldots, a_{\ell}, b, \bar{a}_{1}, \ldots, \bar{a}_{\bar{l}}, \bar{b} \rrbracket,(1 \leqslant i \leqslant l+\bar{l}+2)$, satisfy

(1) $q_{l} \in\left[e^{k_{1}^{*}-1}, e^{k_{1}^{*}}\right], k_{1}^{*} \in\left[k_{1}-\ln M, k_{1}\right], p_{l} / q_{l} \in I, b \geqslant R_{1} / 2$;

(2) $q_{l+\bar{l}+1} \in\left[e^{k_{2}^{*}-1}, e^{k_{2}^{*}}\right], k_{2}^{*} \in\left[k_{2}-\ln M, k_{2}\right], p_{\bar{l}} / q_{\bar{l}} \in I, \bar{b} \geqslant R_{2} / 2$

(3) $k_{2}^{*}>k_{1}^{*}$ (this is where (3.10) is used).

We claim that

$$
\begin{gathered}
\llbracket a_{1}, \ldots, a_{\ell}, b \rrbracket \subset \mathcal{C}_{k_{1}}(I), \\
b \leqslant e^{k_{2}^{*}-k_{1}^{*}+1}, \\
\llbracket \bar{a}_{1}, \ldots, \bar{a}_{\bar{\ell}}, \bar{b} \rrbracket \subset \bigcup_{|r| \leqslant 3} \mathcal{C}_{k_{2}-k_{1}+r-\ln b}\left([0,1], R_{2}\right) .
\end{gathered}
$$

(3.11) follows from (1). Next, $e^{k_{2}^{*}} \geqslant q_{l+1} \geqslant b q_{l} \geqslant b e^{k_{1}^{*}-1}$ proving (3.12). To prove (3.13), let $\bar{p}_{i} / \bar{q}_{i}, 1 \leqslant i \leqslant \bar{\ell}+2$, be the principal convergents of (every) $\bar{\alpha} \in \llbracket b, \bar{a}_{1}, \ldots, \bar{a}_{\bar{\ell}}, \bar{b} \rrbracket$. By Proposition 3.10, $q_{l+1+\bar{l}}=q_{l-1} \bar{p}_{\bar{l}+1}+q_{l} \bar{q}_{\bar{l}+1}$, whence $q_{l} \bar{q}_{\bar{l}+1} \leqslant q_{l+1+\bar{l}} \leqslant 2 q_{l} \bar{q}_{\bar{l}+1}$. Since $q_{l} \in\left[e^{k_{1}^{*}-1}, e^{k_{1}^{*}}\right]$ and $q_{l+\bar{l}+1} \in\left[e^{k_{2}^{*}-1}, e^{k_{2}^{*}}\right]$,

$$
e^{k_{2}^{*}-k_{1}^{*}-2} \leqslant \frac{q_{l+1+\bar{l}}}{2 q_{l}} \leqslant \bar{q}_{\bar{l}+1} \leqslant \frac{q_{l+1+\bar{l}}}{q_{l}} \leqslant e^{k_{2}^{*}-k_{1}^{*}+1} .
$$

Next, let $\widetilde{p}_{i} / \widetilde{q}_{i}(1 \leqslant i \leqslant \bar{l})$ denote the principal convergents of (every) $\widetilde{\alpha} \in \llbracket \bar{a}_{1}, \ldots, \bar{a}_{\bar{\ell}}, \bar{b} \rrbracket$. Then $\frac{\bar{p}_{\bar{l}+1}}{\bar{q}_{\bar{l}+1}}=1 /\left(b+\frac{\widetilde{p}_{\bar{l}}}{\widetilde{q}_{\bar{l}}}\right)$, so $\bar{q}_{\bar{l}+1}=b \widetilde{q}_{\bar{l}}+\widetilde{p}_{\bar{l}}$, whence $b \widetilde{q}_{\bar{l}} \leqslant \bar{q}_{\bar{l}+1} \leqslant(b+1) \widetilde{q}_{\bar{l}}$. Thus $\widetilde{q}_{\bar{l}} \in\left[(b+1)^{-1} \bar{q}_{\bar{l}+1}, b^{-1} \bar{q}_{\bar{l}+1}\right]$. It follows that the $\bar{l}$-th principal convergent of every $\widetilde{\alpha} \in \llbracket \bar{a}_{1}, \ldots, \bar{a}_{\bar{\ell}}, \bar{b} \rrbracket$ satisfies

$$
\widetilde{q}_{\bar{l}} \in\left[e^{k_{2}^{*}-k_{1}^{*}-3-\ln b}, e^{k_{2}^{*}-k_{1}^{*}+1-\ln b}\right] .
$$

It is now easy to see (3.13).

By $(3.13), \mathcal{A}_{k_{1}}(I) \cap \mathcal{A}_{k_{2}}(I) \cap I \subset \bigcup_{|r| \leqslant 3} \biguplus_{[\underline{a}, b] \subset \mathcal{C}_{k_{1}}(I)} \biguplus_{\left[\underline{a}^{\prime}, b^{\prime}\right] \subset \mathcal{C}_{k_{2}-k_{1}+r-\ln b}([0,1])} \llbracket \underline{a}, b, \underline{a}^{\prime}, b \rrbracket$. Now arguing as in the proof of (3.9) and using (3.8) we obtain

$$
\begin{aligned}
& \operatorname{mes}\left(\mathcal{A}_{k_{1}}(I) \cap \mathcal{A}_{k_{2}}(I) \cap I\right) \\
& \leqslant \sum_{\substack{k_{i}^{*} \in\left[k_{i}-\ln M, k_{i}\right] \\
i=1,2 ;|r| \leqslant 3}} \sum_{n \in\left[e^{k_{1}^{*}-1}, e^{\left.k_{1}^{*}\right]}\right.} \sum_{\substack{\operatorname{gcd}(m, n)=1 \\
m / n \in I}} \sum_{b=\left[R_{1} / 2\right]}^{\left[\exp \left(k_{2}^{*}-k_{1}^{*}+1\right)\right]} \frac{2 G \operatorname{mes}\left(\mathcal{C}_{k_{2}-k_{1}+r-\ln b}\left([0,1], R_{2}\right)\right)}{n^{2} b(b+1)} \\
& \leqslant \frac{\text { const mes }(I)}{R_{1} R_{2}} .
\end{aligned}
$$

(3.16) uses the estimate $\operatorname{mes}\left(\mathcal{C}_{k_{2}-k_{1}+r-\ln b}\left([0,1], R_{2}\right)\right)=O\left(1 / R_{2}\right)$ which is also valid when $k_{2}-k_{1}+r-\ln b$ is small, provided we choose $M$ large enough so that the asymptotic in Proposition 3.9 holds for all $N>M$ with $I=[0,1]$. See the proof of $(3.9)$. 
Combining (3.16) with (3.7), we find that under (3.10) $\mathcal{A}_{k_{i}}(I)$ are $D$-quasi-independent for sufficiently large $D$, proving Claim 3 .

Claims 2 and 3 allow us to apply Sullivan's Borel-Cantelli Lemma (Proposition 3.7). We obtain $\delta=\delta(M)$ s.t. for every interval $I \subset[\varepsilon, 1-\varepsilon], \operatorname{mes}(\mathcal{A} \cap I) \geqslant \delta \operatorname{mes}(I)$. This means that $[\varepsilon, 1-\varepsilon] \backslash \mathcal{A}$ has no Lebesgue density points, and therefore must have measure zero. So $\mathcal{A}$ has full measure in $[\varepsilon, 1-\varepsilon]$. Since $\varepsilon$ is arbitrary, $\mathcal{A}$ has full measure.

\section{Proof of Theorem 1.2}

As explained in Section 2, it is enough to prove Theorem 1.2 for $f(x)$ of the form $\sum_{m=1}^{d} b_{m} h\left(x+\beta_{m}\right) \not \equiv 0$ with $h(x)=\{x\}-\frac{1}{2}$. Without loss of generality, $\beta_{i}$ are different and $b_{i} \neq 0$. Notice that

$$
f(x)=-\sum_{m=1}^{d} b_{m} \sum_{j=1}^{\infty} \frac{\sin \left(2 \pi j\left(x+\beta_{m}\right)\right)}{\pi j} .
$$

Therefore $\|f\|_{2}^{2}=\frac{1}{2 \pi^{2}} \sum_{n} \frac{1}{n^{2}} D\left(\beta_{1} n, \ldots, \beta_{d} n\right)$, where $D: \mathbb{T}^{d} \rightarrow \mathbb{R}$ is

$$
D\left(\gamma_{1}, \ldots, \gamma_{d}\right):=\int_{0}^{1}\left[\sum_{m=1}^{d} b_{m} \sin \left(2 \pi\left(y+\gamma_{m}\right)\right)\right]^{2} \mathrm{~d} y .
$$

Since $f \not \equiv 0, D\left(\beta_{1} n, \ldots, \beta_{d} n\right)>0$ for some $n$. Let $\mathfrak{T}$ denote the closure in $\mathbb{T}^{d}$ of $\mathbb{O}:=\left\{\left(\beta_{1} n, \ldots, \beta_{d} n\right) \bmod \mathbb{Z}: n \in \mathbb{Z}\right\}$. This is a minimal set for the translation by $\left(\beta_{1}, \ldots, \beta_{d}\right)$ on $\mathbb{T}^{d}$, so a standard compactness argument shows that for every $\varepsilon_{0}>0$, the set

$$
\mathcal{N}:=\left\{n \in \mathbb{N}: D\left(\beta_{1} n, \ldots, \beta_{d} n\right)>\varepsilon_{0}\right\}
$$

is syndetic: its gaps are bounded. Thus $\mathcal{N}$ has positive lower density.

By Theorem 3.1, if $M$ is sufficiently large then the set $\mathcal{A}:=\mathcal{A}(\mathcal{N}, M)$ has full measure in $\mathbb{T}$. Let

$$
S_{n}(\alpha, x):=\sum_{k=0}^{n-1} f(x+k \alpha) .
$$

The proof of Theorem 1.2 for $f(x)$ above consists of two parts:

Theorem 4.1. - Suppose $\alpha \in \mathcal{A}$, then for a.e. $x \in[0,1)$, there exist $A_{k}(x) \in \mathbb{R}$ and $B_{k}(x), N_{k}(x) \rightarrow \infty$ such that

$$
\frac{S_{n}(\alpha, x)-A_{k}(x)}{B_{k}(x)} \underset{k \rightarrow \infty}{\operatorname{dist}} \longrightarrow \mathrm{U}[0,1] \text {, as } n \sim \mathrm{U}\left(0, \ldots, N_{k}(x)\right) .
$$

Theorem 4.2. - Suppose $\alpha \in \mathcal{A}$, then for a.e. $x \in[0,1)$, there are no $A_{N}(x) \in \mathbb{R}$ and $B_{N}(x) \rightarrow \infty$ such that

$$
\frac{S_{n}(\alpha, x)-A_{N}(x)}{B_{N}(x)} \underset{N \rightarrow \infty}{\operatorname{dist}} \mathrm{U}[0,1], \text { as } n \sim \mathrm{U}(0, \ldots, N) .
$$




\subsection{Preliminaries}

Lemma 4.3. - $S_{q}(\alpha, \cdot): \mathbb{T} \rightarrow \mathbb{R}$ has $d q$ discontinuities.

Proof. - The discontinuities of $S_{q}$ are preimages of discontinuities of $f$ by $R_{\alpha}^{-k}$ with $k=0,1, \ldots, q-1$.

Lemma 4.4. - Let $C:=\sup \left|f^{\prime}\right| \leqslant\left|\sum b_{m}\right|$. If $x^{\prime}, x^{\prime \prime}$ belong to same continuity component of $R_{\alpha}^{r}$ then

$$
\left|S_{r}\left(\alpha, x^{\prime}\right)-S_{r}\left(\alpha, x^{\prime \prime}\right)\right| \leqslant C r\left|x^{\prime}-x^{\prime \prime}\right| .
$$

Proof. - Since $\left|S_{r}^{\prime}\right|=\left|\sum_{k=0}^{r-1} f^{\prime}(x+k \alpha)\right| \leqslant C r$, the restriction of $S_{r}$ to on each continuity component is Lipshitz with Lipshitz constant $C r$.

Lemma 4.5. - There are constants $C_{1}, C_{2}$ such that the following holds. Suppose that $q_{n}$ is a principal denominator of $\alpha$, and $q_{n+1}>c q_{n}$ with $c>1$. Let $\mu_{n}(x):=$ $S_{q_{n}}(\alpha, x)$, then

$$
\operatorname{mes}\left\{x: S_{\ell q_{n}}(\alpha, x)=\ell \mu_{n} \pm C_{1} \frac{\ell^{2}}{c} \text { for } \ell=0, \ldots, k\right\}>1-C_{2} \frac{k}{c} .
$$

Proof. - If $x$ and $x+\ell q_{n} \alpha$ belong to the same continuity interval of $R_{\alpha}^{q_{n}}$ for all $\ell=0, \ldots, k$ then we have by Lemma 4.4 that for $\ell \leqslant k$

$$
\begin{aligned}
& \left|S_{\ell q_{n}}(\alpha, x)-\ell \mu_{n}\right| \leqslant \sum_{j=0}^{\ell-1}\left|S_{q_{n}}\left(\alpha, x+j q_{n} \alpha\right)-S_{q_{n}}(\alpha, x)\right| \leqslant C q_{n} \sum_{j=0}^{\ell-1}\left\|j q_{n} \alpha\right\| \\
& \qquad \frac{C q_{n}}{q_{n+1}} \sum_{j=0}^{\ell-1} j \leqslant \frac{C_{1} \ell^{2}}{c}, \quad \text { where } C_{1}:=C / 2 .
\end{aligned}
$$

Therefore if $S_{\ell q_{n}}(\alpha, x) \neq \ell \mu_{n} \pm C_{1} \frac{\ell^{2}}{c}$ for some $\ell=0, \ldots, k$, then there must exist $0 \leqslant \ell \leqslant k$ s.t. $x, R_{\alpha}^{\ell q_{n}}(x)$ are separated by a discontinuity of $S_{q_{n}}(\alpha, \cdot)$. Since $\operatorname{dist}\left(x, R_{\alpha}^{\ell q_{n}}(x)\right) \leqslant \ell / q_{n+1}, x$ must belong to a ball with radius $k / q_{n+1}$ centered at a discontinuity of $S_{q_{n}}(\alpha, \cdot)$. By Lemma 4.3 , there are $d q_{n}$ discontinuities, so the measure of such points is less than $d q_{n}\left(\frac{2 k}{q_{n+1}}\right) \leqslant \frac{2 d k}{c}$. The lemma follows with $C_{2}:=2 d$.

LEMmA 4.6. - There is a constant $C_{3}=C_{3}\left(b_{1}, \ldots, b_{d}\right)$ s.t. for every $n \geqslant 1$ and $\alpha=\left[0 ; a_{1}, a_{2}, \ldots\right], \max \left\{\left|S_{r}(\alpha, x)\right|: 0 \leqslant r \leqslant q_{n}-1\right\} \leqslant C_{3}\left(a_{0}+\cdots+a_{n-1}\right)$.

Proof. - Let $r=\sum_{j=0}^{n-1} \mathfrak{b}_{j} q_{j}$ denote the Ostrowski expansion of $r$. Recall that this means that $0 \leqslant \mathfrak{b}_{j} \leqslant a_{j}$ and $\mathfrak{b}_{j}=a_{j} \Rightarrow \mathfrak{b}_{j-1}=0$. So

$$
S_{r}=\sum_{k=0}^{\mathfrak{b}_{n-1}-1} S_{q_{n-1}} \circ R_{\alpha}^{q_{n-1} k}+\sum_{k=0}^{\mathfrak{b}_{n-2}-1} S_{q_{n-2}} \circ R_{\alpha}^{q_{n-2} k}+\cdots+\sum_{k=0}^{\mathfrak{b}_{0}-1} S_{q_{0}} \circ R_{\alpha}^{q_{0} k} .
$$

By the Denjoy-Koksma inequality $\left|S_{r}\right| \leqslant \sum \mathfrak{b}_{j} \mathrm{~V}(f) \leqslant \mathrm{V}(f) \sum a_{j}$ where $\mathrm{V}(f) \leqslant 2 \sum b_{i}$ is the total variation of $f$ on $\mathbb{T}$.

LEMmA 4.7. - There exist positive constants $\varepsilon_{1}, \varepsilon_{2}$ such that for every $\alpha$ irrational, if $q_{n}$ is a principal denominator of $\alpha$ and $q_{n} r_{n} \in \mathcal{N}$ with $r_{n} \leqslant M$ then $\operatorname{mes}\left\{x:\left|S_{q_{n}}(\alpha, x)\right| \geqslant \varepsilon_{1}\right\} \geqslant \varepsilon_{2}$. 
Proof. - We follow an argument from [Bec94]. Suppose $q_{n}$ is a principal denominator of $\alpha$ and $q_{n} r_{n} \in \mathcal{N}$ for some $r_{n} \leqslant M$. Let $N=q_{n} r_{n}$. Since $f(x)=$ $-\sum_{m=1}^{d} b_{m} \sum_{j=1}^{\infty} \frac{\sin \left(2 \pi j\left(x+\beta_{m}\right)\right)}{\pi j}$, for each $j \in \mathbb{N}$

$$
\left\|S_{N}(\alpha, \cdot)\right\|_{L^{2}}^{2} \geqslant \frac{1}{\pi^{2} j^{2}} \int_{0}^{1}\left(\sum_{m=1}^{d} b_{m} \sum_{k=0}^{N-1} \sin \left(2 \pi j\left(x+k \alpha+\beta_{m}\right)\right)\right)^{2} \mathrm{~d} x .
$$

Using the identities $\sum_{k=1}^{N} \sin (y+k x)=\frac{\cos (y+x / 2)-\cos (y+(2 N+1) x / 2)}{2 \sin (x / 2)}$ and $\cos A-$ $\cos B=2 \sin \left(\frac{A+B}{2}\right) \sin \left(\frac{B-A}{2}\right)$ we find that

$$
\begin{aligned}
\left\|S_{N}(\alpha, \cdot)\right\|_{L^{2}}^{2} & \geqslant\left(\frac{\sin (\pi N j \alpha)}{\pi j \sin (\pi j \alpha)}\right)^{2} \int_{0}^{1}\left(\sum_{m=1}^{d} b_{m} \sin \left(2 \pi\left(j x+j \frac{(N-1) \alpha}{2}\right)+2 \pi j \beta_{m}\right)\right)^{2} \mathrm{~d} x \\
& =\left(\frac{\sin (\pi N j \alpha)}{\pi j \sin (\pi j \alpha)}\right)^{2} \int_{0}^{1}\left(\sum_{m=1}^{d} b_{m} \sin \left(2 \pi\left(y+j \beta_{m}\right)\right)\right)^{2} \mathrm{~d} y \\
& =\left(\frac{\sin (\pi N j \alpha)}{\pi j \sin (\pi j \alpha)}\right)^{2} D\left(j \beta_{1}, \ldots, j \beta_{m}\right) \quad \text { with } D \text { as in }(4.1) .
\end{aligned}
$$

We now take $j=N=r_{n} q_{n}$. The first term is bounded below because $\|N \alpha\| \leqslant$ $M\left\|q_{n} \alpha\right\| \leqslant \frac{M}{q_{n+1}} \leqslant \frac{M}{a_{n+1} q_{n}} \leqslant \frac{M^{2}}{a_{n+1} N}=o\left(\frac{1}{N}\right)$, so $\frac{\sin \left(\pi N^{2} \alpha\right)}{\pi N \sin (\pi N \alpha)} \underset{n \rightarrow \infty}{\longrightarrow} \pi^{-1}$. The second term is bounded below by $\varepsilon_{0}$, because $N=q_{n} r_{n} \in \mathcal{N}$. It follows that for all $n$ large enough, $\left\|S_{r_{n} q_{n}}(\alpha, \cdot)\right\|_{2}>\sqrt{\varepsilon_{0}} / 2 \pi$.

For any $L^{2}$-function $\varphi$ and any $\hat{\varepsilon}>0$,

$$
\|\varphi\|_{L^{2}}^{2} \leqslant\|\varphi\|_{L^{\infty}}^{2} \operatorname{mes}\{x:|\varphi(x)| \geqslant \hat{\varepsilon}\}+\hat{\varepsilon}^{2} .
$$

Hence $\operatorname{mes}\{x:|\varphi(x)| \geqslant \hat{\varepsilon}\} \geqslant \frac{\|\varphi\|_{L^{2}}^{2}-\hat{\varepsilon}^{2}}{\|\varphi\|_{L^{\infty}}^{2}}$. We just saw that for all $n$ large enough, $\left\|S_{r_{n} q_{n}}(\alpha, \cdot)\right\|_{2}>\sqrt{\varepsilon_{0}} / 2 \pi$, and by the Denjoy-Koksma inequality $\left\|S_{r_{n} q_{n}}(\alpha, \cdot)\right\|_{L^{\infty}} \leqslant$ $M \mathrm{~V}(f)$. So for some $\hat{\varepsilon}>0$ and for all $n$ large enough, $\operatorname{mes}\left\{x:\left|S_{r_{n} q_{n}}(\alpha, x)\right|>\hat{\varepsilon}\right\} \geqslant \hat{\varepsilon}$.

Looking at the inequality $\left|S_{r_{n} q_{n}}(\alpha, x)\right| \leqslant \sum_{k=0}^{r_{n}-1}\left|S_{q_{n}}\left(\alpha, x+k q_{n} \alpha\right)\right|$, we see that if $\left|S_{r_{n} q_{n}}(\alpha, x)\right| \geqslant \hat{\varepsilon}$, then $\left|S_{q_{n}}\left(\alpha, x+k q_{n} \alpha\right)\right| \geqslant \hat{\varepsilon} / M$ for some $0 \leqslant k \leqslant M-1$. So for all $n$ large enough, $\operatorname{mes}\left\{x:\left|S_{q_{n}}(\alpha, x)\right|>\hat{\varepsilon} / M\right\} \geqslant \hat{\varepsilon} / M$.

\subsection{Proof of Theorem 4.1}

Let $\Omega^{*}(\alpha)$ be the set of $x$ where the conclusion of Theorem 4.1 holds. $\Omega^{*}(\alpha)$ is $R_{\alpha}$-invariant and it is measurable by Lemma A.1 in the appendix. Therefore to show that $\Omega^{*}(\alpha)$ has full measure, it suffices to show that it has positive measure.

Suppose $\alpha \in \mathcal{A}$ and let $n_{k} \uparrow \infty$ be a sequence satisfying (3.1) with $\mathcal{N}$ given by (4.2). There is no loss of generality in assuming that

$$
\frac{a_{n_{k}+1}}{a_{0}+\cdots+a_{n_{k}}}>k^{3} .
$$

So $q_{n_{k}+1}>k^{3} L_{k} q_{n_{k}}$, where $L_{k}:=a_{0}+\cdots+a_{n_{k}}$. 
Recall that $\mu_{n_{k}}(x)=S_{q_{n_{k}}}(\alpha, x)$. For all $k$ sufficiently large, there is a set $A_{k}$ of measure at least $\varepsilon_{2} / 2$ such that for all $x \in A_{k}$,

$$
\begin{gathered}
S_{\ell q_{n_{k}}}(\alpha, x)=\ell\left(\mu_{n_{k}}(x) \pm \frac{C_{1} \ell}{k^{3} L_{k}}\right) \text { for all } \ell=0,1, \ldots, k L_{k}, \\
\left|\mu_{n_{k}}(x)\right| \geqslant \varepsilon_{1}
\end{gathered}
$$

This is because Lemma 4.5 says that the total measure of $x$ for which (4.4) fails is $O\left(1 / k^{2}\right)$ while $(4.5)$ holds on the set of measure $\varepsilon_{2}$ by Lemma 4.7.

It follows that mes $\left(\bigcap_{n>1} \bigcup_{k>n} A_{k}\right) \geqslant \varepsilon_{2} / 2$. Therefore there exists $x$ which belongs to infinitely many $A_{k}$. After re-indexing $n_{k}$, we may assume that (4.4), (4.5) are satisfied for all $k \in \mathbb{N}$. Henceforth, we fix such an $x$ and work with this $x$. Let

$$
N_{k}(x):=k L_{k} q_{n_{k}}, \quad B_{k}(x):=k L_{k}\left|\mu_{n_{k}}(x)\right|, \quad A_{k}(x):=\frac{1}{2}\left(\operatorname{sgn}\left(\mu_{n_{k}}(x)\right)-1\right) B_{k} .
$$

Any $n \leqslant N_{k}$ can be written uniquely in the form

$$
n=l(n) q_{n_{k}}+r(n) \quad \text { with } \quad 0 \leqslant l(n) \leqslant k L_{k} \text { and } 0 \leqslant r(n)<q_{n_{k}} .
$$

It is easy to see that $\frac{l(n)}{k L_{k}} \underset{k \rightarrow \infty}{\stackrel{\text { dist }}{\longrightarrow}} \mathrm{U}[0,1]$ as $n \sim \mathrm{U}\left(1, \ldots, N_{k}\right)$.

Writing $S_{n}(\alpha, x)=S_{l(n) q_{n_{k}}}(\alpha, x)+S_{r(n)}\left(\alpha, x+\alpha l(n) q_{n_{k}}\right)$ we obtain from (4.4) and Lemma 4.6 that

$$
S_{n}(\alpha, x)=l(n) \mu_{n_{k}}(x)+O\left(L_{k}\right) .
$$

So $\frac{S_{n}(x)}{B_{k}}$ is asymptotically uniform on $[0,1]$ when $\mu_{n_{k}}>0$, and asymptotically uniform on $[-1,0]$ when $\mu_{n_{k}}<0$. So $\frac{S_{n}(x)-A_{k}}{B_{k}} \underset{k \rightarrow \infty}{\longrightarrow} \mathrm{U}[0,1]$, as $n \sim \mathrm{U}\left(1, \ldots, N_{k}(x)\right)$.

\subsection{Proof of Theorem 4.2}

Let $\Omega(\alpha)$ denote the set of $x \in \mathbb{T}:=\mathbb{R} / \mathbb{Z}$ for which there are $B_{N}(x) \rightarrow \infty$ and $A_{N}(x) \in \mathbb{R}$ s.t.

$$
\frac{S_{n}(\alpha, x)-A_{N}(x)}{B_{N}(x)} \underset{N \rightarrow \infty}{\operatorname{dist}} \longrightarrow \mathrm{U}[0,1], \text { as } n \sim \mathrm{U}(1, \ldots, N) .
$$

$\Omega(\alpha)$ is measurable, and $A_{n}(\cdot), B_{n}(\cdot)$ can be chosen to be measurable on $\Omega(\alpha)$, see the appendix. Assume by way of contradiction that mes $[\Omega(\alpha)] \neq 0$ for some $\alpha \in \mathcal{A}$.

$\Omega(\alpha)$ is invariant under $R_{\alpha}(x)=x+\alpha \bmod 1$ on $\mathbb{T}:=\mathbb{R} / \mathbb{Z}$. Since $R_{\alpha}$ is ergodic, and $\Omega(\alpha)$ is measurable, mes $[\Omega(\alpha)]=1$.

Since $\alpha \in \mathcal{A}$, there is an increasing sequence $n_{k}$ satisfying (3.1) where $\mathcal{N}$ is given by (4.2). We can choose $n_{k}$ so that $q_{n_{k}} r_{n_{k}} \in \mathcal{N}$ for $r_{n_{k}} \leqslant M$, and $a_{n_{k}+1}>k^{3} L_{k}$ where $L_{k}:=a_{0}+\cdots+a_{n_{k}}$. In particular, $q_{n_{k}+1}>k^{3} L_{k} q_{n_{k}}$.

Recall that $\mu_{n_{k}}(x):=S_{q_{n_{k}}}(\alpha, x)$. By Lemma 4.7 we can choose $x$ such that for infinitely many $k,\left|\mu_{n_{k}}(x)\right| \geqslant \varepsilon_{1}$. We will suppose that $\mu_{n_{k}}(x)>0$ for infinitely many $k$; the case where $\mu_{n_{k}}(x)<0$ for infinitely many $k$ is similar.

Claim 4. - It is possible to assume without loss of generality that $\left\|B_{q_{n_{k}}}\right\|_{\infty}:=$ $\sup _{x \in \Omega(\alpha)}\left|B_{q_{n_{k}}}(x)\right| \leqslant 3 C_{3} L_{k}$ for all $k$ where $C_{3}$ is the constant from Lemma 4.6. 
Proof. - We claim that for every $x$ with (4.6), $B_{q_{n_{k}}}(x) \leqslant 3 C_{3} L_{k}$ for all $k$ large enough. Otherwise, by Lemma 4.6, there are infinitely many $k$ s.t. $B_{q_{n_{k}}}(x)>$ $3 \max \left\{\left|S_{r}(\alpha, x)\right|: r=0, \ldots, q_{n_{k}-1}\right\}$, whence $\left|S_{n}(\alpha, x) / B_{q_{n_{k}}}\right| \leqslant \frac{1}{3}$ for all $0 \leqslant n \leqslant$ $q_{n_{k}}-1$. In such circumstances, (4.6) does not hold (the spread is not big enough).

Since $B_{q_{n_{k}}}(x) \leqslant 3 C_{3} L_{k}$ for all $k$ large enough, there is no harm in replacing $B_{{q_{n}}}(x)$ in (4.6) by $\min \left\{B_{q_{n_{k}}}(x), 3 C_{3} L_{k}\right\}$.

Claim 5. - Fix $D>C=\left|\sum b_{m}\right|$, and let $E_{k}$ denote the set of $x \in \Omega(\alpha)$ s.t. $S_{r}(\alpha, x)=S_{r}\left(\alpha, R_{\alpha}^{\ell q_{n_{k}}}(x)\right) \pm \frac{D \ell}{q_{n_{k}}+1}$ for all $0 \leqslant \ell \leqslant B_{q_{n_{k}}}(x), 0 \leqslant r<q_{n_{k}}-1$. Then $\operatorname{mes}\left(E_{k}^{c}\right) \leqslant C_{4} k^{-3}$.

Proof. - If $x \notin E_{k}$, then there are $0 \leqslant \ell \leqslant B_{q_{n_{k}}}(x), 0 \leqslant r<q_{n_{k}}-1$ s.t.

$$
\left|S_{r}(\alpha, x)-S_{r}\left(\alpha, x+\ell q_{n_{k}} \alpha\right)\right| \geqslant \frac{D \ell}{q_{n_{k}+1}} .
$$

By Lemma 4.4, $\{x\},\left\{x+\ell q_{n_{k}} \alpha\right\}$ are separated by a singularity of $S_{r}(\alpha, \cdot)$. So $x$ belongs to a ball of radius $2\left\|\ell q_{n_{k}} \alpha\right\|$ centered at one of the $d q_{n_{k}}$ discontinuities of $S_{q_{n_{k}}}(\alpha, \ldots)$. Thus $\operatorname{mes}\left(E_{k}^{c}\right) \leqslant d q_{n_{k}} \cdot 2\left\|\ell q_{n_{k}} \alpha\right\|$. Now $\left\|\ell q_{n_{k}} \alpha\right\| \leqslant \ell\left\|q_{n_{k}} \alpha\right\| \leqslant \frac{\left\|B_{q_{n_{k}}}\right\|_{\infty}}{q_{n_{k}+1}} \leqslant$ $\frac{3 C_{3} L_{k}}{q_{n_{k}+1}} \leqslant \frac{3 C_{3}}{k^{3} q_{n_{k}}}$ by our choice of $n_{k}$. So mes $\left(E_{k}^{c}\right) \leqslant C_{4} / k^{3}$ with $C_{4}:=6 d C_{3}$.

Claim 6. - Let $F_{k}$ denote the set of $x \in \Omega(\alpha)$ s.t.

$$
S_{\ell_{q_{k}}}(\alpha, x)=\ell\left(\mu_{n_{k}}(x) \pm \frac{C_{1} \ell}{k^{3} L_{k}}\right) \text { for all } 0 \leqslant \ell \leqslant B_{q_{n_{k}}}(x) .
$$

Then $\operatorname{mes}\left(F_{k}^{c}\right) \leqslant C_{5} k^{-2}$.

Proof. - This follows from Lemma 4.5.

By Claims 5 and 6, and a Borel-Cantelli argument, for a.e. $x$ there is $k_{0}(x)$ s.t. $x \in E_{k} \cap F_{k}$ for all $k \geqslant k_{0}(x)$.

Suppose $k \geqslant k_{0}(x)$, and let $N_{k}:=q_{n_{k}} B_{q_{n_{k}}}(x)$. Every $0 \leqslant n \leqslant N_{k}-1$ can be uniquely represented as $n=\ell q_{n_{k}}+r$ with $0 \leqslant \ell \leqslant B_{q_{n_{k}}}(x)-1$ and $0 \leqslant r \leqslant q_{n_{k}}-1$. Using the bound $\left\|B_{q_{n_{k}}}\right\|_{\infty}=O\left(L_{k}\right)$, we find:

$$
\begin{aligned}
& \frac{S_{n}(\alpha, x)-A_{q_{n_{k}}}(x)}{B_{q_{n_{k}}}(x)} \\
& =\frac{S_{\ell q_{n_{k}}}(\alpha, x)}{B_{q_{n_{k}}}(x)}+\frac{S_{r}\left(\alpha, R_{\alpha}^{\ell q_{n_{k}}} x\right)-A_{q_{n_{k}}}(x)}{B_{q_{n_{k}}}(x)} \\
& =\frac{S_{\ell q_{n_{k}}}(\alpha, x)}{B_{q_{n_{k}}}(x)}+\frac{S_{r}(\alpha, x)-A_{q_{n_{k}}}(x)+o(1)}{B_{q_{n_{k}}}(x)}, \quad \text { because } x \in E_{k} \\
& =\frac{\ell\left(\mu_{n_{k}}(x)+o(1)\right)}{B_{q_{n_{k}}}(x)}+\frac{S_{r}(\alpha, x)-A_{q_{n_{k}}}(x)+o(1)}{B_{q_{n_{k}}}(x)}, \quad \text { because } x \in F_{k} .
\end{aligned}
$$

If $n \sim \mathrm{U}\left(0, \ldots, N_{k}-1\right)$, then $\ell, r$ are independent random variables, $\ell \sim \mathrm{U}(0, \ldots$, $\left.B_{q_{n_{k}}}(x)-1\right)$ and $r \sim \mathrm{U}\left(0, \ldots, q_{n_{k}}-1\right)$. Thus the distribution of $\frac{\ell\left(\mu_{n_{k}}(x)+o(1)\right)}{B_{q_{n_{k}}}(x)}$ is 
close to $\mathrm{U}\left[0, \mu_{n_{k}}(x)\right]$, and the distribution of $\frac{S_{r}(\alpha, x)-A_{q_{n_{k}}}(x)+o(1)}{B_{q_{n_{k}}}(x)}$ converges to $\mathrm{U}[0,1]$ (because $x \in \Omega$ ).

Taking a subsequence such that $\mu_{n_{k}}(x) \rightarrow \bar{\varepsilon}>\varepsilon_{1}$ we see that the random variables $\frac{S_{n}(\alpha, x)-A_{q_{n_{k}}}(x)}{B_{q_{n_{k}}}(x)}$, where $n \sim \mathrm{U}\left(0, \ldots, n_{k}-1\right)$, converge in distribution to the sum of two independent uniformly distributed random variables. This contradicts to (4.6), because the sum of two independent uniform random variables is not uniform.

\section{Appendix A. Measurability concerns}

Let $\Omega(\alpha)$ denote the set of $x \in \mathbb{T}:=\mathbb{R} / \mathbb{Z}$ such that for some $B_{N}(x) \rightarrow \infty$ and $A_{N}(x) \in \mathbb{R}, \frac{S_{n}(\alpha, x)-A_{N}(x)}{B_{N}(x)} \underset{N \rightarrow \infty}{\stackrel{\text { dist }}{\longrightarrow}} \mathrm{U}[0,1]$, as $n \sim \mathrm{U}(1, \ldots, N)$ and let $\Omega^{*}(\alpha)$ denote the set of $x \in \mathbb{T}$ such that along a subsequence $N_{k}(x)$ there exist some $B_{N_{k}}(x) \rightarrow \infty$ and $A_{N_{k}}(x) \in \mathbb{R}, \frac{S_{n}(\alpha, x)-A_{N_{k}}(x)}{B_{N_{k}}(x)} \underset{k \rightarrow \infty}{\stackrel{\text { dist }}{\longrightarrow}} \mathrm{U}[0,1]$, as $n \sim \mathrm{U}\left(1, \ldots, N_{k}\right)$. We make no assumptions on the measurability of $A_{N}, B_{N}, N_{k}$ as functions of $x$. The purpose of this section is to prove:

LEMmA A.1. $-\Omega(\alpha)$ and $\Omega^{*}(\alpha)$ are measurable.

The crux of the argument is to show that $A_{N}(x), B_{N}(x)$ can be replaced by measurable functions, defined in terms of the percentiles of the random quantities $S_{n}(x, \alpha)$, $n \sim \mathrm{U}(1, \ldots, N)$.

Recall that given $0<t<1$, the upper and lower $t$-percentiles of a random variable $X$ are defined by

$$
\begin{aligned}
& \chi^{+}(X, t):=\inf \{\xi: \operatorname{Pr}(X \leqslant \xi)>t\} \\
& \chi^{-}(X, t):=\sup \{\xi: \operatorname{Pr}(X \leqslant \xi)<t\} \quad(0<t<1) .
\end{aligned}
$$

Notice that $\operatorname{Pr}\left(X \leqslant \chi^{+}(X, t)\right) \geqslant t, \operatorname{Pr}\left(X<\chi^{-}(X, t)\right) \leqslant t$, and $\operatorname{Pr}\left(\chi^{-}(X, t)<X<\right.$ $\left.\chi^{+}(X, t)\right)=0$. In case $X$ is non-atomic (i.e. $\operatorname{Pr}(X=a)=0$ for all $a$ ), we can say more:

Lemma A.2. - Suppose $X$ is a non-atomic real valued random variable, fix $0<t<1$ and let $\chi_{t}^{ \pm}:=\chi^{ \pm}(X, t)$, then

(a) $\operatorname{Pr}\left(X<\chi_{t}^{+}\right)=t$ and $\operatorname{Pr}\left(X<\chi_{t}^{-}\right)=t$;

(b) $\forall \varepsilon>0, \operatorname{Pr}\left(\chi_{t}^{-}-\varepsilon<X<\chi_{t}^{-}\right), \operatorname{Pr}\left(\chi_{t}^{+}<X<\chi_{t}^{+}+\varepsilon\right)$ are positive;

(c) $\exists t_{1}<t_{2}$ s.t. $\chi_{t_{1}}^{-}<\chi_{t_{2}}^{+}$and $\chi_{t_{1}}^{-}, \chi_{t_{2}}^{+}$have the same sign.

Proof. - Since $X$ is non-atomic, $\operatorname{Pr}\left(X<\chi_{t}^{+}\right)=\operatorname{Pr}\left(X \leqslant \chi_{t}^{+}\right) \geqslant t$ and $\operatorname{Pr}(X<$ $\left.\chi_{t}^{-}\right) \leqslant t$. If $\chi_{t}^{+}=\chi_{t}^{-}$, part (a) holds. If $\chi_{t}^{+}>\chi_{t}^{-}$then for all $h>0$ small enough $\chi_{t}^{-}+h<\chi_{t}^{+}-h$ whence

$$
\begin{aligned}
0 \leqslant \operatorname{Pr}\left(\chi_{t}^{-}<X<\chi_{t}^{+}\right)=\lim _{h \rightarrow 0^{+}} \operatorname{Pr}\left(\chi_{t}^{-}+h<X<\chi_{t}^{+}-h\right) \\
\quad=\lim _{h \rightarrow 0^{+}} \operatorname{Pr}\left(X<\chi_{t}^{+}-h\right)-\lim _{h \rightarrow 0^{+}} \operatorname{Pr}\left(X \leqslant \chi_{t}^{-}+h\right) \leqslant t-t=0 .
\end{aligned}
$$

Necessarily $\lim _{h \rightarrow 0^{+}} \operatorname{Pr}\left(X<\chi_{t}^{+}-h\right)=t$ and $\lim _{h \rightarrow 0^{+}} \operatorname{Pr}\left(X \leqslant \chi_{t}^{-}+h\right)=t$, which gives us $\operatorname{Pr}\left(X<\chi_{t}^{+}\right)=t$ and $\operatorname{Pr}\left(X<\chi_{t}^{+}\right)=\operatorname{Pr}\left(X \leqslant \chi_{t}^{+}\right)=t$. 
For (b) assume by contradiction that $\operatorname{Pr}\left(\chi_{t}^{-}-\varepsilon<X<\chi_{t}^{-}\right)=0$, then for all $\chi_{t}^{-}-\varepsilon<\xi<\chi_{t}^{-}, \operatorname{Pr}(X \leqslant \xi)=\operatorname{Pr}\left(X<\chi_{t}^{-}\right)=t$, whence $\chi_{t}^{-} \leqslant \chi_{t}^{-}-\varepsilon$, a contradiction. Similarly, $\operatorname{Pr}\left(\chi_{t}^{+}<X<\chi_{t}^{+}+\varepsilon\right)=0$ is impossible.

To prove (c) note that since $X$ is non-atomic, either $\operatorname{Pr}(X>0)$ or $\operatorname{Pr}(X<0)$ is positive. Assume w.l.o.g. that $\operatorname{Pr}(X>0) \neq 0$. By non-atomicity, there are positive $a<b$ s.t. $\operatorname{Pr}(X \in(0, a)) \neq 0$ and $\operatorname{Pr}(X \in(a, b)) \neq 0$. Take $t_{1}:=\operatorname{Pr}(X<a)$ and $t_{2}:=\operatorname{Pr}(X<b)$.

From now on fix a non-atomic random variable $Y$, and choose $0<t_{1}<t_{2}<1$ as in Lemma A.2(c) s.t. $\chi^{-}\left(Y, t_{1}\right)<\chi^{+}\left(Y, t_{2}\right)$ and $\operatorname{sgn}\left(\chi^{-}\left(Y, t_{1}\right)\right)=\operatorname{sgn}\left(\chi^{+}\left(Y, t_{2}\right)\right)$.

Lemma A.3. - Let $S_{N}$ be (possibly atomic) random variables s.t. for some $A_{N} \in \mathbb{R}$ and $B_{N} \rightarrow \infty, \frac{S_{N}-A_{N}}{B_{N}} \underset{N \rightarrow \infty}{\stackrel{\text { dist }}{\longrightarrow}} Y$. Then $\frac{S_{N}-A_{N}^{*}}{B_{N}^{*}} \underset{N \rightarrow \infty}{\stackrel{\text { dist }}{\longrightarrow}} Y$, where $A_{N}^{*}, B_{N}^{*}$ are the unique solution to

$$
\left\{\begin{array}{l}
A_{N}^{*}+B_{N}^{*} \chi^{-}\left(Y, t_{1}\right)=\chi^{-}\left(S_{N}, t_{1}\right) \\
A_{N}^{*}+B_{N}^{*} \chi^{+}\left(Y, t_{2}\right)=\chi^{+}\left(S_{N}, t_{2}\right) .
\end{array}\right.
$$

Proof. - Without loss of generality, $\chi^{-}\left(Y, t_{1}\right), \chi^{+}\left(Y, t_{2}\right)$ are both positive.

We need the following fact (which is not automatic since $S_{N}$ are allowed to be atomic):

$$
\lim _{N \rightarrow \infty} \operatorname{Pr}\left(S_{N}<\chi^{-}\left(S_{N}, t\right)\right)=t \text { for all } 0<t<1 .
$$

Indeed, given $\varepsilon>0$, let $\xi_{N}:=B_{N} \chi^{-}(Y, t-\varepsilon)+A_{N}$, then

$$
\operatorname{Pr}\left(S_{N}<\xi_{N}\right)=\operatorname{Pr}\left(\frac{S_{N}-A_{N}}{B_{N}}<\chi^{-}(Y, t-\varepsilon)\right) \underset{N \rightarrow \infty}{\longrightarrow} \operatorname{Pr}\left(Y<\chi^{-}(Y, t-\varepsilon)\right)=t-\varepsilon,
$$

by Lemma A.2 (a). So for all $N$ large enough, $\xi_{N} \leqslant \chi^{-}\left(S_{N}, t\right)$, whence $\lim \inf \operatorname{Pr}\left(S_{N}<\right.$ $\left.\chi^{-}\left(S_{N}, t\right)\right) \geqslant \lim \operatorname{Pr}\left(S_{N}<\xi_{N}\right)=t-\varepsilon$. Since $\varepsilon$ is arbitrary, $\lim \inf \operatorname{Pr}\left(S_{N}<\right.$ $\left.\chi^{-}\left(S_{N}, t\right)\right) \geqslant t$. The other inequality $\lim \sup \operatorname{Pr}\left(S_{N}<\chi^{-}\left(S_{N}, t\right)\right) \leqslant t$ is clear since $\operatorname{Pr}\left(S_{N}<\chi^{-}\left(S_{N}, t\right)\right) \leqslant t$ for all $N$.

With (A.2) proved, we proceed to prove that

$$
\frac{A_{N}^{*}-A_{N}}{B_{N}} \underset{N \rightarrow \infty}{\longrightarrow} 0 \text { and } \quad \frac{B_{N}^{*}}{B_{N}} \underset{N \rightarrow \infty}{\longrightarrow} 1 \text {. }
$$

It will then be obvious that $\frac{S_{N}-A_{N}}{B_{N}} \underset{N \rightarrow \infty}{\stackrel{\text { dist }}{\longrightarrow}} Y$ implies $\frac{S_{N}-A_{N}^{*}}{B_{N}^{*}} \underset{N \rightarrow \infty}{\stackrel{\text { dist }}{\longrightarrow}} Y$.

Define two affine transformations, $\varphi_{N}(t)=\frac{t-A_{N}}{B_{N}}$ and $\varphi_{N}^{*}(t)=\frac{t-A_{N}^{*}}{B_{N}^{*}}$. Notice that $\left(\varphi_{N}^{*}\right)^{-1}(t)=A_{N}^{*}+B_{N}^{*} t$, so $\left(\varphi_{N}^{*}\right)^{-1}\left(\chi^{-}\left(Y, t_{1}\right)\right)=\chi^{-}\left(S_{N}, t_{1}\right)$, by (A.1). Since $B_{N}^{*}=\frac{\chi^{+}\left(S_{N}, t_{2}\right)-\chi^{-}\left(S_{N}, t_{1}\right)}{\chi^{+}\left(Y, t_{2}\right)-\chi^{-}\left(Y, t_{1}\right)}>0, \varphi_{N}^{*}$ is increasing. By $(\mathrm{A} .2), \operatorname{Pr}\left(\varphi_{N}^{*}\left(S_{N}\right)<\chi^{-}\left(Y, t_{1}\right)\right)=$ $\operatorname{Pr}\left(S_{N}<\chi^{-}\left(S_{N}, t_{1}\right)\right) \underset{N \rightarrow \infty}{\longrightarrow} t_{1}$. So

$$
\begin{aligned}
t_{1} & =\lim _{N \rightarrow \infty} \operatorname{Pr}\left(\varphi_{N}^{*}\left(S_{N}\right)<\chi^{-}\left(Y, t_{1}\right)\right) \\
& =\lim _{N \rightarrow \infty} \operatorname{Pr}\left(\varphi_{N}\left(S_{N}\right)<\varphi_{N}\left[\left(\varphi_{N}^{*}\right)^{-1}\left(\chi^{-}\left(Y, t_{1}\right)\right)\right]\right) \\
& =\lim _{N \rightarrow \infty} \operatorname{Pr}\left(\varphi_{N}\left(S_{N}\right)<\frac{B_{N}^{*}}{B_{N}}\left(\chi^{-}\left(Y, t_{1}\right)+\frac{A_{N}^{*}-A_{N}}{B_{N}}\right)\right) .
\end{aligned}
$$


We claim that this implies that

$$
\liminf _{N \rightarrow \infty} \frac{B_{N}^{*}}{B_{N}}\left(\chi^{-}\left(Y, t_{1}\right)+\frac{A_{N}^{*}-A_{N}}{B_{N}}\right) \geqslant \chi^{-}\left(Y, t_{1}\right) .
$$

Otherwise, $\exists \varepsilon$ s.t. $\liminf _{N \rightarrow \infty} \frac{B_{N}^{*}}{B_{N}}\left(\chi^{-}\left(Y, t_{1}\right)+\frac{A_{N}^{*}-A_{N}}{B_{N}}\right)<\chi^{-}\left(Y, t_{1}\right)-\varepsilon$, so

$$
\begin{aligned}
t_{1} & =\liminf _{N \rightarrow \infty} \operatorname{Pr}\left(\varphi_{N}\left(S_{N}\right)<\frac{B_{N}^{*}}{B_{N}}\left(\chi^{-}\left(Y, t_{1}\right)+\frac{A_{N}^{*}-A_{N}}{B_{N}}\right)\right) \\
& \leqslant \liminf _{N \rightarrow \infty} \operatorname{Pr}\left(\varphi_{N}\left(S_{N}\right)<\chi^{-}\left(Y, t_{1}\right)-\varepsilon\right)=\operatorname{Pr}\left(Y<\chi^{-}\left(Y, t_{1}\right)-\varepsilon\right) \\
& =\operatorname{Pr}\left(Y<\chi^{-}\left(Y, t_{1}\right)\right)-\operatorname{Pr}\left(\chi^{-}\left(Y, t_{1}\right)-\varepsilon \leqslant Y<t_{1}\right) \\
& <t_{1}, \text { by Lemma A.2(a) and (b). }
\end{aligned}
$$

Similarly, one shows that

$$
\limsup _{N \rightarrow \infty} \frac{B_{N}^{*}}{B_{N}}\left(\chi^{+}\left(Y, t_{2}\right)+\frac{A_{N}^{*}-A_{N}}{B_{N}}\right) \leqslant \chi^{+}\left(Y, t_{2}\right) .
$$

It remains to see that (A.4) and (A.5) imply (A.3). First we divide (A.4) by (A.5) to obtain

$$
\limsup _{N \rightarrow \infty} \frac{\chi^{-}\left(Y, t_{1}\right)+\frac{A_{N}^{*}-A_{N}}{B_{N}}}{\chi^{+}\left(Y, t_{2}\right)+\frac{A_{N}^{*}-A_{N}}{B_{N}}} \geqslant \frac{\chi^{-}\left(Y, t_{1}\right)}{\chi^{+}\left(Y, t_{2}\right)} .
$$

Since $x \mapsto \frac{a+x}{b+x}$ is strictly decreasing on $[0, \infty)$ when $a>b>0$, this implies that

$$
\limsup _{N \rightarrow \infty} \frac{A_{N}^{*}-A_{N}}{B_{N}} \leqslant 0 .
$$

Looking at (A.4), and recalling that $\chi^{-}\left(Y, t_{1}\right)>0$, we deduce that

$$
\liminf _{N \rightarrow \infty} \frac{B_{N}^{*}}{B_{N}} \geqslant 1
$$

Next we look at the difference of (A.4) and (A.5) and obtain

$$
\limsup _{N \rightarrow \infty} \frac{B_{N}^{*}}{B_{N}}\left(\chi^{+}\left(Y, t_{2}\right)-\chi^{-}\left(Y, t_{1}\right)\right) \leqslant \chi^{+}\left(Y, t_{2}\right)-\chi^{-}\left(Y, t_{1}\right),
$$

whence $\lim \sup \left(B_{N}^{*} / B_{N}\right) \leqslant 1$. Together with (A.7), this proves that $B_{N}^{*} / B_{N} \underset{N \rightarrow \infty}{\longrightarrow}$. Substituting this in (A.4), gives $\liminf \frac{A_{N}^{*}-A_{N}}{B_{N}} \geqslant 0$, which, in view of (A.6), implies that $\frac{A_{N}^{*}-A_{N}}{B_{N}} \underset{N \rightarrow \infty}{\longrightarrow} 0$. This completes the proof of (A.3), and with it, the lemma.

Proof of Lemma A.1. - We begin with the measurability of $\Omega(\alpha)$.

Let $S_{N}(x)$ denote the random variable equal to $S_{n}(\alpha, x)$ with probability $\frac{1}{N}$ for each $1 \leqslant n \leqslant N$.

We will apply Lemma A.3 with $Y:=\mathrm{U}[0,1], S_{N}=S_{n}(x)$ and (say) $t_{1}:=\frac{1}{3}, t_{2}:=\frac{2}{3}$. It says that

$$
\Omega(\alpha)=\left\{x \in \mathbb{T}: \frac{S_{N}(x)-A_{N}^{*}(x)}{B_{N}^{*}(x)} \underset{N \rightarrow \infty}{\stackrel{\operatorname{dist}}{\longrightarrow}} \mathrm{U}[0,1]\right\},
$$


where $A_{N}^{*}(x)$ and $B_{N}^{*}(x)$ are the unique solutions to (A.1). Since the percentiles of $S_{N}(x)$ are measurable as functions of $x, A_{N}^{*}(x), B_{N}^{*}(x)$ are measurable as functions of $x$.

We claim that $\Omega(\alpha)=\Omega_{1}(\alpha) \cap \Omega_{2}(\alpha)$ where

$$
\begin{aligned}
& \Omega_{1}(\alpha):=\bigcap_{\ell=1}^{\infty} \bigcup_{M=1}^{\infty} \bigcap_{N=M+1}^{\infty}\left\{x \in \mathbb{T}: \frac{1}{N} \sum_{n=1}^{N} 1_{(2, \infty)}\left(\left|\frac{S_{n}(\alpha, x)-A_{N}^{*}(x)}{B_{N}^{*}(x)}\right|\right)<\frac{1}{\ell}\right\} \\
& \Omega_{2}(\alpha)=\bigcap_{t \in \mathbb{Q} \backslash\{0\}}\left\{x \in \mathbb{T}: \lim _{N \rightarrow \infty} \frac{1}{N} \sum_{n=1}^{N} e^{i t\left(\frac{S_{n}(\alpha, x)-A_{N}^{*}}{B_{N}^{*}(x)}\right)}=\mathbb{E}\left(e^{i t Y}\right)\right\} .
\end{aligned}
$$

This will prove the lemma, since the measurability of $A_{N}^{*}(\cdot), B_{N}^{*}(\cdot)$ implies the measurability of $\Omega_{i}(\alpha)$.

If $x \in \Omega(\alpha)$ then

$$
x \in \Omega_{1}(\alpha), \quad \text { because } \operatorname{Pr}\left[\left|\frac{S_{N}(x)-A_{N}^{*}}{B_{N}^{*}}\right|>2\right] \underset{N \rightarrow \infty}{\longrightarrow} 0,
$$

and $\quad x \in \Omega_{2}\left(\alpha_{2}\right), \quad$ because $\mathbb{E}\left(e^{i t\left(\frac{S_{N}(x)-A_{N}^{*}}{B_{N}^{*}}\right)}\right) \underset{N \rightarrow \infty}{\longrightarrow} \mathbb{E}\left(e^{i t Y}\right)$ pointwise.

Conversely, if $x \in \Omega_{1}(\alpha) \cap \Omega_{2}(\alpha)$ then it is not difficult to see that

$$
\mathbb{E}\left(e^{i t\left(\frac{S_{N}(x)-A_{N}^{*}}{B_{N}^{*}}\right)}\right) \underset{N \rightarrow \infty}{\longrightarrow} \mathbb{E}\left(e^{i t Y}\right)
$$

for all $t \in \mathbb{R}$. So $x \in \Omega(\alpha)$ by Lévy's continuity theorem. Thus $\Omega(\alpha)=\Omega_{1}(\alpha) \cap \Omega_{2}(\alpha)$, whence $\Omega(\alpha)$ is measurable.

The proof that $\Omega^{*}(\alpha)$ is measurable is similar. Enumerate $\mathbb{Q} \backslash\{0\}=\left\{t_{n}: n \in \mathbb{N}\right\}$, then $\alpha \in \Omega^{*}(\alpha)$ iff for every $\ell \in \mathbb{N}$ there exist $M \in \mathbb{N}$ s.t. for some $N>M$

$$
\begin{aligned}
& \frac{1}{N} \sum_{n=1}^{N} 1_{(2, \infty)}\left(\left|\frac{S_{n}(\alpha, x)-A_{N}^{*}(x)}{B_{N}^{*}(x)}\right|\right)<\frac{1}{\ell} \\
& \left|\mathbb{E}\left(e^{i t_{n}\left(\frac{S_{N}(x)-A_{N}^{*}(x)}{B_{N}^{*}(x)}\right)}\right)-\mathbb{E}\left(e^{i t_{n} Y}\right)\right|<\frac{1}{\ell} \text { for all } n=1, \ldots, \ell .
\end{aligned}
$$

These are measurable conditions, because $A_{N}^{*}(\cdot), B_{N}^{*}(\cdot)$ are measurable. So $\Omega^{*}(\alpha)$ is measurable.

\section{BIBLIOGRAPHY}

[ADDS15] Artur Avila, Dmitry Dolgopyat, Eduard Duryev, and Omri Sarig, The visits to zero of a random walk driven by an irrational rotation, Isr. J. Math. 207 (2015), no. 2, 653-717. $\uparrow 128$

[ADU93] Jon Aaronson, Manfred Denker, and Mariusz Urbański, Ergodic theory for Markov fibred systems and parabolic rational maps, Trans. Am. Math. Soc. 337 (1993), no. 2, 495-548. $\uparrow 136$

[AK82] Jon Aaronson and Michael Keane, The visits to zero of some deterministic random walks, Proc. Lond. Math. Soc. 44 (1982), no. 3, 535-553. ^128 
[Bec94] József Beck, Probabilistic Diophantine approximation. I. Kronecker sequences, Ann. Math. 140 (1994), no. 1, 109-160. $\uparrow 140$

[Bec10]_, Randomness of the square root of 2 and the giant leap. I, Period. Math. Hung. 60 (2010), no. 2, 137-242. $\uparrow 128$

[Bec11] Randomness of the square root of 2 and the giant leap. II, Period. Math. Hung. 62 (2011), no. 2, 127-246. $\uparrow 128$

[BU18] Michael Bromberg and Corinna Ulcigrai, A temporal Central Limit Theorem for realvalued cocycles over rotations, Ann. Inst. Henri Poincaré, Probab. Stat. 54 (2018), no. 4, 2304-2334. $\uparrow 128$

[CK76] Jean-Pierre Conze and Michael Keane, Ergodicité d'un flot cylindrique, Séminaire de Probabilités, I (Univ. Rennes, Rennes, 1976), Université Rennes, 1976, Exp. no. 5, 7 pages. $\uparrow 128$

[DS17a] Dmitry Dolgopyat and Omri Sarig, Quenched and annealed temporal limit theorems for circle rotations, 28 pages, preprint, 2017. $\uparrow 129,130$

[DS17b] _ Temporal distributional limit theorems for dynamical systems, J. Stat. Phys. 166 (2017), no. 3-4, 680-713. $\uparrow 128,130$

[DS18] _ Asymptotic windings of horocycles, Isr. J. Math. 228 (2018), no. 1, 119-176. $\uparrow 128$

[DV86] Harold G. Diamond and Jeffrey D. Vaaler, Estimates for partial sums of continued fraction partial quotients, Pac. J. Math. 122 (1986), no. 1, 73-82. $\uparrow 131$

[HW08] Godfrey Harold Hardy and Edward Maitland Wright, An introduction to the theory of numbers, sixth ed., Oxford University Press, 2008, Revised by D. R. Heath-Brown and J. H. Silverman, With a foreword by Andrew Wiles. $\uparrow 133$

[Jar29] Vojtech Jarník, Zur metrischen Theorie der diophantischen Approximationen, Prace Mat.-Fiz. 36 (1929), no. 1, 91-106. ^128

[Khi24] Alexander Ya. Khintchine, Einige Sätze über Kettenbrüche, mit Anwendungen auf die Theorie der Diophantischen Approximationen, Math. Ann. 92 (1924), no. 1-2, 115-125. $\uparrow 128$

[Khi63] _ Continued fractions, P. Noordhoff, Ltd., 1963, translated by Peter Wynn. $\uparrow 128$, 132,136

[PS17] Elliot Paquette and Younghwan Son, Birkhoff sum fluctuations in substitution dynamical systems, Ergodic Theory Dyn. Syst. (2017). $\uparrow 128$

[Sch78] Klaus Schmidt, A cylinder flow arising from irregularity of distribution, Compos. Math. 36 (1978), no. 3, 225-232. ^128

[Sul82] Dennis Sullivan, Disjoint spheres, approximation by imaginary quadratic numbers, and the logarithm law for geodesics, Acta Math. 149 (1982), no. 3-4, 215-237. $\uparrow 132$

Manuscript received on 14th March 2018, revised on 24th October 2018, accepted on 21st November 2018.

Recommended by Editor S. Gouëzel. Published under license CC BY 4.0.

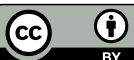

This journal is a member of Centre Mersenne.

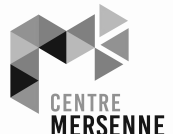


Dmitry DOLGOPYAT

Department of Mathematics

University of Maryland at College Park

College Park, MD 20742 (USA)

dmitry@math.umd.edu

Omri SARIG

Faculty of Mathematics and Computer Science

The Weizmann Institute of Science

234 Herzl Street

7610001 Rehovot (Israel)

omsarig@gmail.com 